\title{
An Alternative Way to Make Knowledge Sharing Work in Online Communities? The Effects of Hidden Knowledge Facilitators
}

\author{
Jason Li-Ying, ${ }^{1}$ Zhinan Zhang, ${ }^{2}$ and Qing Long ${ }^{3}$ \\ ${ }_{1}^{1}$ Technical University of Denmark, Denmark, ${ }^{2}$ Shanghai Fiaotong University, China, and ${ }^{3}$ Hunan \\ Land Enterprise Consulting Company, China
}

ABSTRAGT Some firms use hidden knowledge facilitators (HKFs) to facilitate knowledge sharing among employees within intrafirm online communities. These firms hope for enhanced knowledge sharing outcomes within their organizations without letting employees know that HKFs exist. Yet, the extent to which HKFs' interventions are effective remains unknown to researchers and managers. Built on the knowledge sharing (KS) literature, this study explores the unique roles of HKFs as moderators between a company and its employees. We develop several hypotheses to test the impact of the quantity and quality of HKFs' online interventions on several KS outcomes. By analyzing log data of a Chinese corporation's online R\&D community, we find that (1) the quantity of HKFs' intervention has a mostly positive impact on KS outcomes; (2) the quality of HKFs' intervention has a mixed impact on several KS outcomes, depending on which aspect of quantity is considered; and (3) the quality of HKFs' intervention also moderates the positive impact of the quantity of HKFs' intervention in different ways on different intended KS outcomes. This study makes a clear contribution to the literature on knowledge sharing and knowledge facilitation by demonstrating the impact of HKFs on KS outcomes in a Chinese context.

KEYWORDS bandwagon effect, content analysis, divergent and convergent thinking, knowledge facilitator, knowledge sharing, online communities

\section{INTRODUGTION}

Researchers have long recognized that knowledge sharing (KS) among employees allows organizations to exploit and capitalize on knowledge-based resources, which contribute to knowledge application, innovation, and ultimately the competitive advantage of the organization (Jackson, Chuang, Harden, Jiang, \& Joseph, 2006). To pursue the alleged benefits of KS, many organizations have deployed virtual communities to foster online KS among employees (Lee \& Choi, 2003;

Corresponding author: Jason Li-Ying (yinli@dtu.dk)

C 2018 The International Association for Chinese Management Research 
Wellman, Salaff, Dimitrova, Garton, Gulia, \& Haythornthwaite, 1996). The literature on online communities suggests a rational choice perspective (Grandori, 1997), which puts a premium on understanding the attributes of users (Jeppesen \& Fredriksen, 2006), aligning the motivations of participation and contribution (Shah, 2006), and deploying formal and informal governance mechanisms within online communities (Li-Ying \& Salomo, 2013). The underlying logic embedded in such a stream of literature implies that as long as users with appropriate attributes in online communities are motivated according to their attributes and corresponding governance is in place, KS will somehow occur (Cook, 2008).

However, several studies find that some factors may hinder KS behaviors in virtual communities (Chen \& Hung, 2010; Hsu, Ju, Yen, \& Chang, 2007); thus online virtual communities need facilitators as a supporting tool to reduce KS barriers and enhance the effectiveness of KS enablers, such as corporate culture and trust, which eventually cause KS behaviors to actually take place (Ardichvili, 2008). The role of facilitation in online KS communities needs more research attention (Cacciamani, Cesareni, Martini, Ferrini, \& Fujita, 2012) because the literature to date has not clearly addressed the effectiveness of specific supporting tools in online KS communities and the corresponding outcomes for organizations with respect to the KS behaviors of online users (employees) (Chen \& Hung, 2010; Wang \& Noe, 2010). Even worse, the literature on KS is unclear about whether KS behavior creates positive outcomes that benefit organizations (Haas \& Hansen, 2007; Wang \& Noe, 2010).

To contribute to this underdeveloped research area, in this study we focus on the effectiveness of a specific (and a rather unconventional) type of supporting mechanism of online KS virtual communities within an organization: hidden knowledge facilitators. Recently, we have observed some firms starting to use a small number of employees to facilitate $\mathrm{KS}$ in intrafirm online $\mathrm{R} \& \mathrm{D}$ communities without revealing their identities as facilitators. These hidden knowledge facilitators $(\mathrm{HKFs})$ are responsible to the firm, which 'hires' them to influence the interactions on the intrafirm online R\&D community in hopes of enhancing KS and innovations. These HKFs actively participate in the online KS communities and may create a bandwagon effect for other regular community members to follow (Leibenstein, 1950) - a phenomenon that is similar to that in consumer psychology where increasing demand creates more demand (van Herpen, Pieters, \& Zeelenberg, 2009). The intentions of these HKFs are to serve the company as a whole, and employees who seek new knowledge in particular, by facilitating online KS within the firm. The firm's management imposes a job mandate on these HKFs to facilitate online R\&D communities by boosting online KS behaviors, while HKFs' job mandate and performance objectives are hidden from regular employees, who might not be willing to share their specific knowledge with colleagues, should they know the online traffic of discussion is somehow 'manipulated' by HKFs. The special status and functions of HKFs in firms' online R\&D communities provide us with a perfect research context for 
investigating how effective HKFs are at making their online contributions to yield positive KS outcomes for their organization.

In this study, we ask a research question: to what extent does the quantity and quality of HKFs' online interventions effectively lead to positive knowledge sharing outcomes? The answer to this question is important for managers to understand how to use HKFs as a supporting mechanism to enhance intraorganizational KS. To understand the effectiveness of $\mathrm{HKF}_{\mathrm{S}}$, several theories, including user communities in innovation management, the bandwagon effect in sociology, and consumer psychology, are relevant. However, none of them alone sufficiently explains the effect of HKFs. In our research design, we focus on the KS literature and take the phenomenon of $\mathrm{HKF}$ s as a specific case to highlight the effectiveness of a supporting mechanism for online $\mathrm{KS}$, while drawing on insights from several relevant theories to build argumentation for hypothesis development. Using log data from an intrafirm online R\&D KS community in a large Chinese company, we use a netnographic method based on extensive content analysis to test several hypotheses about the relationships between the quantity and quality of HKFs' online interventions and KS outcomes among employees. In Table 1, we summarize the research design and conceptual model, on which several hypotheses will be developed, and the related methodology will be detailed in the next sections.

We pay special attention to three concrete KS outcomes: enhanced online discussion (reflected in the length of discussion), problem resolution, and convergent and divergent thinking. This is because these measurable KS outcomes, respectively, correspond closely to the three major $\mathrm{KS}$ facilitation functions suggested theoretically by prior studies (Cabrera \& Cabrera, 2002), namely, encouraging communication, decreasing $\mathrm{KS}$ cost and increasing $\mathrm{KS}$ benefit, and increasing perceived efficacy of contributors.

This study makes a direct contribution to the literature on KS on virtual platforms in general and the design of intrafirm online knowledge community in particular. First, in this study, we clearly show to what extent HKFs' interventions affect organizational KS outcomes. Thus, a missing link in the literature on KS regarding whether KS behavior creates positive outcomes that benefit organizations (Haas \& Hansen, 2007; Wang \& Noe, 2010) is established with empirical evidence. Second, in a more nuanced sense, this study pinpoints the importance of considering both the quantity and the quality of HKFs' interventions when an organization designs its online KS communities. We suggest managers at any organization deploying or considering deploying HKFs in their $\mathrm{KS}$ intranet to pay attention to the joint properties of quantity and quality in the work of HKFs.

\section{THEORETICAL BACKGROUND}

\section{Knowledge Sharing}

An organization's capacity to share knowledge among its employees and apply that shared knowledge to perform important activities is increasingly seen as a vital 
Table 1. Research design and conceptual model

\begin{tabular}{ll} 
Research question & \multicolumn{1}{c}{ Research methods } \\
\hline $\begin{array}{l}\text { To what extent the quantity and } \\
\text { quality of HKFs online inter- }\end{array}$ & $\begin{array}{l}\text { Quantitative methods (hypoth- } \\
\text { esis testing using regression }\end{array}$ \\
$\begin{array}{l}\text { ventions will effectively lead to } \\
\text { potential positive knowledge }\end{array}$ & $\begin{array}{l}\text { analysis based on content ana- } \\
\text { lysis of log data) }\end{array}$ \\
sharing outcomes? &
\end{tabular}

Key concepts to be explored/key relationships to be tested

Independent variables:

HKFs' online intervention

1. Quantity of intervention

2. Quality of intervention

Dependent variables:

KS outcomes

1. Length of discussion

2. Solutions found

3. Divergent and convergent discussion

source of competitive advantage (Damodaran \& Olphert, 2000; Jackson et al., 2006). Knowledge sharing refers to the provision of task information and individual expertise to help and collaborate with others to solve problems, develop new ideas, or implement policies or procedures. This process takes place via different means of communications, including the traditional paper format or various electronic formats (Cummings, 2004; Haas \& Hansen, 2007). Recently, KS via digital means such as online discussion forums and communities has become extremely popular among various organizations (Cacciamani et al., 2012; Ma \& Yuen, 2011).

KS is believed to benefit organizations. In a recent review of KS, Wang and Noe (2010) found research has shown that KS makes a potentially positive impact on firms' performance in terms of reductions in production costs, rapid completion of new product development projects, effective team performance, innovation capabilities, and consequent increases in sales and revenue from new products and services. However, as KS also bears costs and barriers both within and across organizations, obtaining and using knowledge from other parts of a firm does not necessarily guarantee improved firm performance (Haas \& Hansen, 2005). Therefore, recent studies have emphasized that scholars need to move beyond studying the facilitating conditions for KS and pay more attention to examining whether and how facilitated KS eventually leads to positive organizational outcomes (Haas \& Hansen, 2007).

The literature also recognizes that sharing codified and tacit knowledge within and across organizations by either electronic or interpersonal means (Bordia, Irmer, \& Abusah, 2006) does not take place by itself, because KS intention and behavior are influenced by organizational culture and climate (Taylor \& Wright, 2004), personal attributes (Cabrera, Collins, \& Salgado, 2006), and incentives (Shah, 2006; Wasko \& Faraj, 2005) - a complex system that needs management support and facilitation (Kulkarni, Ravindran, \& Freeze, 2006). Therefore, intervention mechanisms and management support are needed to facilitate KS (Cabrera et al., 2006).

(C) 2018 The International Association for Chinese Management Research 


\section{Motivating and Bandwagon Effect: The Need for Knowledge Sharing Facilitation}

The need for KS facilitation within organizations and on virtual KS communities in particular can be understood based on at least two streams of literature: one is on how to motivate online KS participation and contribution, and the other is about creating a bandwagon effect.

With respect to the first, when an organization establishes and launches an online KS platform, there is no guarantee that employees will find it interesting to participate and share knowledge on it; in fact, there is a potential lack of motivation to do so. Many case studies have demonstrated that people are motivated to join online KS communities for very different reasons (Dahlander \& Magnusson, 2005; Dutton, 2008; Nambisan, 2002; Nambisan \& Baron, 2007; Shah, 2006; von Hippel \& von Krogh, 2003; West \& O’Mahony, 2008). While some find contributing to a particular community intrinsically interesting, others are extrinsically motivated to seek solutions to their specific needs (Jeppesen \& Frederiksen, 2006; Shah, 2006). A firm normally needs to foster community interactions among differently motivated users, create a sense of belonging, and show strong commitment from top management (Ma \& Yuen, 2011). Providing appropriate incentives for altering or manipulating one's motivations is a key function of $\mathrm{KS}$ facilitation (Sundaramurthy \& Lewis, 2003; Walsh \& Seward, 1990).

With respect to the second stream of literature, when a small number of employees can be motivated to start sharing knowledge, ideally many others will follow, creating a so-called bandwagon effect. As a non-functional demand in economics, the bandwagon effect refers to the extent to which the demand for a commodity increases because other people are buying the same commodity (Leibenstein, 1950). Early studies in economics on demand theory and theories in consumer psychology have noticed non-additivity in consumer demand, which has links to herd behavior (Banerjee, 1992; Bikhchandani, Hirshleifer, \& Welch, 1998; Corneo \& Jeanne, 1997) and the psychological theories of conformity and social influence (Asch, 1955; Baron, Vandello, \& Brunsman, 1996). Bandwagon effects occur when consumers follow the behavior of others, either because they want to get 'into the swim of things', or conform with the people they wish to associate with (Berger \& Heath, 2007; Escalas \& Bettman, 2005), or because they believe that the choice behavior of others reveals a product's uniqueness (van Herpen et al., 2009) or superior quality (Huang \& Chen, 2006; Kardes, Posavac, \& Cronley, 2004), which they cannot afford to go without. The behavior of others thus provides additional clues as the consumer creates a mental shortcut used as a judgment rule for making quick evaluations. This is the so-called bandwagon heuristic (Sundar, 2007; Sundar, Oeldorf-Hirsch, $\& \mathrm{Xu}, 2008)$. In other cases, the presence and observable act of an expert might trigger the expert heuristic, which leads directly to positive evaluations of an expert's statement without scrutiny of its content. Often, the joint forces of the bandwagon heuristic and the expert heuristic create a bandwagon effect on actors' social behavior. 
In the context of KS within organizations, as organizational, cultural, and structural hurdles prevail and prevent employees from actively sharing knowledge, various kinds of business practices are deployed to create a bandwagon effect that can benefit a firm. For instance, knowledge facilitators in an online education and training environment have received some research attention, particularly with respect to the role of online tutor support around facilitator styles and metacognition (Cacciamani et al., 2012). Among other things, online facilitator support contributes to the educational success of online education activities, as the facilitators play their roles as instructors, facilitators, and moderators (Cesareni et al., 2008). The basic premise in this stream of research is that a moderated online community is preferable to a non-moderated one, because the bandwagon effect can be created and utilized by online community facilitators (Wise, Hamman, \& Thorson, 2006).

\section{Knowledge Sharing Facilitations and KS Outcomes}

A vast majority of the research on $\mathrm{KS}$ has focused on what factors motivate or hinder KS behavior without addressing whether KS behaviors actually lead to positive outcomes for organizations (e.g., Gagné, 2009; Pee \& Min, 2017; Reinholt, Pedersen, \& Foss, 2011). In other words, the literature has provided us with rich insights on why people share (or do not share) knowledge within and across organizations, but whether KS behaviors actually bring any advantage to organizations is still to some extent unclear (Haas \& Hansen, 2007; Naim \& Lenkar, 2017; Wang \& Noe, 2010). To address this research gap, we adopt the KS intervention mechanisms suggested by Cabrera and Cabrera (2002) and focus on how HKFs' intervention functions attain positive outcomes (if any) for the KS community, which in turn benefit the organization.

Cabrera and Cabrera (2002) lay a profound theoretical foundation for KS facilitation functions: (1) to promote group identity and personal responsibility by encouraging communication; (2) to decrease KS cost and increase sharing benefit; and (3) to increase perceived efficacy of contributors. It can be argued that the deployment of an online R\&D community platform itself and the corresponding HR policy oriented toward rewarding KS on the intranet are themselves unique KS management practices (interventions) that help decrease KS cost and increase KS benefit. Nevertheless, firms need to design and implement other forms of interventions to facilitate KS, such as awards (Dahlander \& Magnusson, 2005), regular support from community managers and senior management (Nambisan, 2002; Nambisan \& Baron, 2007), and protocols and guidelines (Gilbert \& Dabbagh, 2005). To our knowledge, while the KS facilitation mechanisms suggested by Cabrera and Cabrera (2002) have received much research attention, they have rarely been used to test the relationships between interventions and KS outcomes directly.

Despite all the useful means of facilitating $\mathrm{KS}$ that are addressed in prior studies, the phenomenon of HKFs is new to researchers and practitioners. 
Compared to conventional online KS facilitators, HKFs are believed to play their facilitating roles differently for at least two reasons. First, whether and to what extent expert heuristics can be created and effective is questionable, as HKFs' identities are unknown to online community members. Second, HKFs are appointed by the firm and their job responsibilities of boosting $\mathrm{KS}$ in the intrafirm online community are unknown to regular community members (other employees). Their acts do cause other regular online community members to make decisions (for example, uploading documents, sharing experiences, posting messages, and participating in collaborative innovation) that they would not otherwise make. This creates a natural experiment with a condition, under which KS facilitation is deemed as non-existing from a regular online community member's view, removing any potential priming effect on KS behaviors of community members due to the knowledge about being 'facilitated' by non-genuine members. This, in turn, makes the HKFs a perfect case to investigate the effect of KS interventions on KS outcomes. Having noticed the importance and special features of HKFs, we are motivated to investigate the objective impacts of HKFs on KS outcomes in relation to Cabrera and Cabrera's typology of intervention mechanisms so that the established theory in KS facilitation can be consolidated.

\section{HYPOTHESES DEVELOPMENT}

In this study, we chose three indicators of community-level KS outcomes, which are associated with the generic online intervention mechanisms proposed by Cabrera and Cabrera (2002). First, the length of discussion in each thread directly indicates the extent to which information and knowledge are exchanged among online community members (Masters \& Oberprieler, 2004; Nisbet, 2004). This $\mathrm{KS}$ outcome indicator corresponds to the first intervention mechanisms proposed by Cabrera and Cabrera (2002), namely, encouraging communication. The second online intervention mechanism is to decrease $\mathrm{KS}$ cost and increase $\mathrm{KS}$ benefit. This mechanism is fundamentally important because a discussion thread of any length bears communication costs to participating members. If it does not reach a solution to the question or problem, participants in the discussion will deem this form of KS useless and members who did not directly participate in the discussion will get the impression that the $\mathrm{KS}$ community is ineffective. In relation to this mechanism, a straightforward indicator of KS outcome is to evaluate whether a solution is found, or a problem resolved in each discussion thread. A positive result justifies the KS cost and creates a positive sum of KS benefit within the online community. Whether a solution is found or not reflects community-outcome expectations (Hsu et al., 2007). The third intervention mechanism proposed by Cabrera and Cabrera (2002) is to increase the perceived efficacy of contributors. Ideally, HKFs should aim at inducing online community members to make useful, reliable, and creative contributions. The meaningful consequence of doing so at the community level is the observable formation of divergent and 
convergent discussion (Runco \& Acar, 2012) within each discussion thread. ${ }^{[1]}$ This is because convergent thinking among contributors indicates that someone's contribution is useful and collectively considered to be reliable; divergent thinking among contributors indicates that they think from different perspectives to solve the problem. Collective convergent and divergent thinking both have creative potential and are good indicators of effective learning as a result of KS (Dijksterhuis \& Meurs, 2006; Runco, 2007). Table 2 summarizes the generic intervention mechanisms of $\mathrm{KS}$ facilitators and the corresponding choices of KS outcomes.

As far as HKFs' observable interventions are concerned, we notice that HKFs need to constantly monitor the development of opinions and information dissemination on the online community to become effective advocates for KS. Actual online intervention behaviors are concrete actions that are performed by actively posting original discussion topics, responding to other community members' posts, posting knowledge sources, and uploading useful documents.

Online interventions can take various forms, and typically can be observed in one of two ways: by the classification of communication patterns (Adendorff, 2005; Westerski, Dalamagas, \& Iglesias, 2013) or by generically observing the quantity and quality of observations based on content analysis (Bliss \& Lawrence, 2009; Gilbert \& Dabbagh, 2005; Nisbet, 2004). HKFs are a special type of KS facilitators and we have little knowledge on how to classify their intervention patterns. In contrast, observing the quantity and quality of HKFs' interventions can be achieved relatively objectively, and the results may provide a foundation of understanding about their communication patterns for future research. Therefore, we focus on observing the quantity and quality of HKFs' intervention in our research design. First, quantity matters because a silent $\mathrm{HKF}$ will never effectively facilitate KS in the online R\&D community. A straightforward measure of online interaction quantity is, for instance, a count of the number of posts made by community members (Masters \& Oberprieler, 2004). Second, an intervention with low quality might be ineffective because it might be unnoticed or regarded as useless and unreliable. Prior studies have found that the quality of interaction in asynchronous discussion forums has a positive impact on individuals' learning outcomes (Nandi, Hamilton, \& Harland, 2012). In a sense, high quality online intervention reflects the extent to which the information provided in an online post meets or exceeds the expectations of those who receive or use it because it is sufficiently informative and reliable and relatively easy for other community members to refer to and further act upon (Hackman, 1987; Nisbet, 2004). Therefore, both quantity and quality of online interventions are important factors in the managerial objective of making an impact on KS within communities.

Moreover, we argue that when many HKFs coordinate their interventions collectively, it is possible to create a bandwagon heuristic; when a small number of HKFs repeatedly contribute to the online community, they might be seen as experts, creating an expert heuristic. Both heuristics are supposed to facilitate KS. However, the quantity and quality of online interventions need to be 
Table 2. Online intervention mechanisms (Cabrera \& Cabrera, 2002) and corresponding community level KS outcomes

Intervention mechanisms (Cabrera \& Cabrera, 2002)

Encouraging communication (to promote group identity and personal responsibility)

Decrease KS cost and increase KS benefit

Increase perceived efficacy of contributors
Community level KS outcomes

Length of discussion (per thread)

Solution found or not

Divergent and convergent thinking

considered in combination because their joint effects can be more relevant than their separate effects: a large quantity of low quality interventions may create an impression of useless online discussion; a large quantity of high quality intervention may create information overload and confusion (Edmunds \& Morris, 2000); a small quantity of low quality interventions will become completely unnoticed; and a small quantity of high quality interventions will have limited reach within an online community. Therefore, we will develop hypotheses for both separate and joint effects of quantity and quality of HKFs interventions.

\section{Hypotheses}

First, HKFs cannot afford to stand by when there is an inactive discussion forum. To encourage communication in online KS communities, and in turn promote group identity and personal responsibility among community members, HKFs need to actively post online by commenting, suggesting clues to solving problems, introducing new ideas, encouraging feedback, or directly providing sources of information and documents (Nisbet, 2004; Westerski et al., 2013). Like regular online community managers, HKFs need to ensure frequent and timely feedback in the online community to create the feeling of belonging that some employees appreciate (Dahlander \& Magnusson, 2008; Nambisan, 2002; Nambisan \& Baron, 2007). The more interventions as such they make, the longer a discussion will last, allowing more employees to participate and exchange information. In some cases, even if other community members are not initially activated by HKFs' online intervention, the posts made by the HKFs themselves may lengthen the discussion, making it appear more interesting so that the thread may catch other members' attention later on. In this respect, the quantity of HKFs' online interventions are clearly related to the length of discussion as a quantitative indicator of community KS outcome. We thus hypothesize,

\section{Hypothesis 1: The quantity of hidden knowledge facilitators' interventions will be positively} associated with the length of discussion.

When the quality of the interventions is high, online community members are able to learn from HKFs' posts, access the right sources of information and 
knowledge, become inspired, and be more likely to engage in further discussion. High quality HKF interventions can also reduce the time needed by other employees to search for information and learn so that the learning cost of the entire community in general can be reduced (Haas \& Hansen, 2007). This potentially results in timesaving for community members participating in online discussions and sharing what they have learned. In contrast, low quality HKF interventions offer little value for KS and learning, discouraging further participation in discussions by other community members (Correia \& Baran, 2010). Therefore, we argue that high quality $\mathrm{HKF}$ interventions will foster more online $\mathrm{KS}$. We thus hypothesize,

Hypothesis 2: The quality of hidden knowledge facilitators' interventions will be positively associated with the length of discussion.

Furthermore, it is possible that the effects of quantity and quality of $\mathrm{HKF}$ interventions may interact. When several HKFs make high quality online interventions on average in a discussion thread, the solution to a problem is found more quickly and there are fewer opportunities for other community members to participate. The consequence is that a KS circle is completed, and the related knowledge documented, formulated, and institutionalized. Thus, it tends to end a discussion thread sooner. Notably, this interplay between quantity and quality of HKFs' intervention suggests a rapid learning process, indicated by the length of discussion for each thread, but does not suggest inferior quality KS. In fact, HKFs can deliberately shorten an online discussion thread by contributing a number of high quality posts that provide concrete clues to solving the problem. In other words, when HKFs make a number of high quality posts in a thread, the aim of the HKFs is likely to end the discussion by providing a solution as quickly as possible. Our hypothesis is thus:

\section{Hypothesis 3: The positive relationship between the quantity of hidden knowledge facilitators' intervention and the length of discussion will be less evident when the quality of the interventions is high.}

In an online R\&D KS forum, engineers and managers typically ask various kinds of R\&D-related professional questions, as they contend with different work problems and challenges on a daily basis. The intrafirm online $\mathrm{R} \& \mathrm{D}$ forum becomes a natural choice where the entire community can render some help, as long as someone in the community has the knowledge to provide the right solution or guidance to finding the solution. Therefore, it is very important for the knowledge seeker and the community as a whole to recognize that solutions to posted questions are usually found, a positive indicator of a KS outcome at the community level. In a sense, the online R\&D community provides a shortcut for knowledge seekers to find the knowledge within an organization, particularly when high quality online interventions are performed. With respect to any particular question posted online, the more clues and information provided by HKFs, the more likely a solution will be 
found, because a thread with (seemingly) heated discussions catches people's attention and it is more likely that online community members will collectively solve the problem (Jeppesen \& Frederiksen, 2006). Therefore, we hypothesize,

\section{Hypothesis 4: The quantity of hidden knowledge facilitators' interventions will be positively associated with the likelihood of solutions being found.}

In our observation, HFKs can use different approaches when intervening in a discussion thread in order to facilitate employees finding a solution to a posted problem. For instance, they can simply acknowledge the relevance and importance of the problem, help clarify the question, provide some sources of relevant information for people to discuss, verify the usefulness of tips, refer to a specific person who is knowledgeable about the problem, or suggest a solution to the problem directly. These approaches present different levels of quality of interventions, which online community members find useful to varying degrees. When the quality of an HKF's intervention is high, it is sufficiently informative and constructive that the knowledge seeker and other discussion participants can rely on the high-quality posts to either find a feasible path toward a solution based on HKF comments or directly accept a suggested solution. In other cases, HKFs can 'promote' challenging development tasks by making high quality interventions to challenge seekers, making them intrinsically interested and satisfied (Dahlander \& Magnusson, 2005). Thus, HKFs' high quality interventions tend to help employees find solutions. We hypothesize accordingly,

Hypothesis 5: The quality of hidden knowledge facilitators' interventions will be positively associated with the likelihood of solutions being found.

However, when we take both quantity and quality of HKFs' interventions into account, we might have a quite different observation. When an intervention is of high quality with little need for rework, it usually means some concrete information or a possible solution to the problem is suggested for the knowledge seekers to consider and verify. In these cases, a small quantity of high quality interventions will suffice, because if a high-quality approach is used too often, it will present too many concrete information clues for the initial knowledge seekers and follower participants to verify and absorb. A large number of high quality HFKs interventions in turn might make people confused about what the actual and effective solutions are. Here, our expectation is that at high levels of quality, the positive effect of quantity is reduced. Therefore, we have the next hypothesis:

Hypothesis 6: The positive relationship between the quantity of hidden knowledge facilitators' intervention and the likelihood of solutions being found will be less evident when the quality of these interventions is high.

Next, we consider divergent and convergent thinking. These two processes require imaginary and rational cognitive information processing, respectively, 
based on a certain level of useful clues or information (Dijksterhuis \& Meurs, 2006). While divergent thinking is responsible for creating novelty, convergent thinking, which evaluates novel ideas based on knowledge, is an important component of individual creativity (Cropley, 2006). Though conceptually distinguishable, divergent thinking and convergent thinking are two highly integrated parts of creative thinking and they reinforce each other to perform the function of creativity (Runco \& Acar, 2012). On the one hand, without convergent thinking, divergent thinking is useless and meaningless; on the other hand, without divergent thinking, convergent thinking has no ground to build on. These two cognitive processes take place recurrently within the mind. As divergent thinking and convergent thinking are not mutually exclusive, it is possible that an external factor may have an effect on them in the same direction, depending on the context. In our specific case, it depends on the intention/purpose of the HKFs. Note that the HKFs are employed by the firm with a special job mandate and they know about each other. Therefore, their actions of interventions in online threads are highly coordinated. For some issues, HKFs want to inspire regular employees and get as many diversified discussions as possible; for other issues, HKFs might simply want to prevent people from spending too much energy in exploring options by quickly focusing on the right choices of solutions. Bearing this in mind, we develop some hypotheses for the effects of HKFs' online interventions on convergent thinking and divergent thinking separately.

A convergent discussion pattern in online $\mathrm{KS}$ communities serves as a safeguard and an enabler for creative solution development in groups. Thus, convergent discussion can be viewed as an aspect of organizational creativity, a KS outcome that reflects the increased efficacy of online contributors as a whole. For convergent thinking, the quantity of interventions matters mainly due to herd behaviors as a result of bandwagon effects created by HKFs (Leibenstein, 1950). That is, the more HKFs make interventions, the more likely it is that a bandwagon effect will be created, where people seem to agree with each other. This function is similar to ghost customers (ghost bidders) in online stores, where positive feedback from a large number of ghost customers suggests a quality and price advantage of a product, so that actual online customers are influenced by these opinions and make irrational purchases (van Herpen et al., 2009). Following this line of reasoning, we hypothesize,

Hypothesis 7: The quantity of hidden knowledge facilitators' interventions will be positively associated with convergent discussion.

Moreover, when an HKF's post is of low quality, providing little useful information or knowledge that others can use directly or build upon, it will be hard to trigger any further convergent thinking among other community members, because little knowledge is provided to help community members evaluate ideas and potential solutions. In practice, HFKs can foster convergent discussions by 
posting high quality interventions with concrete sources of critical information and knowledge, pathways to solutions, and criteria for judging effectiveness and novelty (Cropley, 2006). HKFs' high quality interventions may also create potential expert heuristics, which also contributes to a bandwagon effect. Therefore, we predict,

Hypothesis 8: The quality of hidden knowledge facilitators' interventions will be positively associated with convergent discussion.

While a bandwagon effect can be achieved through a large quantity of online interventions or some high-quality interventions, a strategy of combining both could potentially backfire. If many HFKs make high quality posts in small numbers (aiming at creating a bandwagon heuristic) or a small number of HFKs repeatedly make high quality posts (aiming at creating an expert heuristic), the result is that too much seemingly useful information and too many feasible pathways to solve problems are presented to the online community. A large quantity of high quality HKF interventions could create information overload and confusion (Edmunds \& Morris, 2000; Eppler \& Mengis, 2004), rather than convergent thinking. Note that this does not necessarily mean that it is impossible for any individual to learn, but for the community at large it will create stress, rather than a foundation for organizational creativity. Therefore, we predict that,

Hypothesis 9: The positive relationship between the quantity of hidden knowledge facilitators' intervention and convergent discussion will be less evident when the quality of these interventions is high.

Divergent thinking is another critical aspect of creativity. Although divergent thinking alone does not guarantee creativity, it is by nature variety-seeking, and acts as the source of novelty, which is then subject to convergent thinking for evaluation (Runco \& Acar, 2012). A divergent discussion in an online community features many ideas, information clues, and alternative pathways to solving problems. Quantity is a double-edged sword - it can be used to create a bandwagon effect to confirm on the right solution so that people's ideas converge, but it can also be used to inspire diversified discussion by 'stirring the pot'. Thus, we expect a large quantity of HKFs' interventions will steer divergent discussions and help diversify the direction of discussions. The more HKFs intervene, the more diversified the discussions are. Therefore, we predict that,

Hypothesis 10: The quantity of hidden knowledge facilitators' interventions will be positively associated with divergent discussion.

As far as the quality of HKFs' intervention is concerned, low quality interventions lack concrete information clues, making them of little use for idea diversification within online discussions. However, if an HKF's post appears to be a clear solution to a problem with little need for rework, indicating a high level of 
intervention quality, then it will be difficult to ignite further divergent thinking in other community members, because a KS and learning circle has been completed within the online discussion. Ideally, KFs' interventions at a medium level of quality will require additional conceptual and practical work from others, either by providing a new viewpoint or referral to a (media) file, document, standard, or knowledgeable person. This inevitably forces people to think and act according to a guided cognitive path through divergent thinking (Basadur, Runco, \& Vega, 2000). Based on these arguments, we suggest the following hypothesis:

Hypothesis 11: The quality of hidden knowledge facilitators' interventions will have an inverted- $U$ shaped relationship with divergent discussion.

Finally, if HKFs' medium quality interventions are the best means of igniting divergent discussion in an online community, then we expect that a large number of HKF interventions will intensify such an effect and make the optimal level of medium level quality more evident. This is because, on an online discussion thread with a small number of $\mathrm{HKF}$ posts, a few medium quality interventions might get full attention once or twice, so that other community members will directly follow the suggestion to seek additional information in order to solve the problem. The effect on diversified opinions will not be that evident. However, if the HKFs make a large number of interventions of varying quality, the inspiring and igniting effect of those with medium quality on divergent thinking will be evident, because the large number of interventions makes it possible for the medium quality ones to stand out. Therefore, we hypothesize,

Hypothesis 12: The inverted U-shaped relationship between the quality of hidden knowledge facilitators' interventions and divergent discussions will be more evident when the quantity of interventions is large.

\section{EMPIRICAL BACKGROUND}

The empirical context for this research is within a Chinese multinational heavy machinery manufacturing company (for confidentiality reasons, it will be referred hereinafter as 'the company'), headquartered in Hunan Province. This company is one of the largest heavy equipment manufacturers in the world, listed on the FT Global 500 and the Forbes Global 2000 indexes. The company is organized into a number of major divisions and subsidiaries, including a concrete pump division, a road construction division, a port machinery division, a mobile crane division, an electric utility company, two heavy machinery subsidiaries, a heavy equipment subsidiary, and a science and technology subsidiary. The company has four international R\&D and manufacturing facilities in India, the USA, Germany, and Brazil. The company puts a premium on R\&D excellence and pursues global leadership in product innovation in its industry. On average, 5-7 percent of the group's annual 
revenue is used for R\&D investment. By the end of 2014, the company had made 8,282 Chinese patent applications and 405 international patent applications.

The company has approximately 90,000 employees worldwide, of which 4,000 R\&D personnel are located in China within about 70 in-house R\&D institutes. The intrafirm online R\&D platform was established in June 2012 and has been operating ever since. It was designed and introduced with the purpose of enhancing KS and innovation among R\&D personnel. In principle, all the company's R\&D personnel in China have access to the online platform as regular users, and several online forums have been formed around topic areas such as hydraulic engineering, mechanical engineering, electrical engineering, material engineering, specific crafts, technology benchmarking, and simulation. These forums form virtual knowledge communities that can be roughly divided into six categories: technological exchanges, RED management, application of tools, product development, knowledge management, and administrative topics.

Since its establishment, the intrafirm online R\&D platform has received increasing attention from top management and R\&D personnel within the company. This has been demonstrated by the fact that the management of all the research institutes has recognized its relevance to the company's strategy and development of employee competence and has participated in discussions in the online forums; and that the average number of monthly visits and viewing time has been consistently increasing. Still, according to the manager of the intrafirm online R\&D platform, it had not yet reached its full effectiveness when the research was conducted: extensive discussions and exchange of knowledge had been relatively concentrated within a dozen $R \& D$ institutes and only a few hundred active R\&D personnel comprised the key online community members. For this reason, the question of how to systematically use HKFs among these forums (communities) to boost KS caught management's attention. Thanks to the support of the senior manager of the online communities, we were given access to multiple data sources related to the intrafirm R\&D online communities and HKFs.

This case company was chosen for a number of specific reasons: first, it has been using $\mathrm{HKF}_{\mathrm{s}}$ to facilitate online $\mathrm{R} \& \mathrm{D}$ communities for a relatively long period, allowing longitudinal observations; second, we are able to get full access to the entire population of HKFs within the company and observe their online log data for a sufficiently long period; and third, the company is representative of large corporations using intranet platforms to facilitate internal $\mathrm{KS}$ in multiple areas of technological R\&D.

Prior to collecting online log data of the community communication, we conducted several semi-structured interviews with key staff members, who are highly representative of different perspectives in the company. The purpose of conducting these pre-study interviews was to have a sound understanding of who the HKFs are and how they function. The interviewees comprised the chief manager of the intrafirm online communities, two official $\mathrm{HKF}_{\mathrm{s}}$, and a regular community member. All interviewees were employees of the company and registered users of the 
online R\&D platform. We did not associate the questions with any established theoretical perspectives on knowledge facilitators, for instance, the mechanisms suggested by Cabrera and Cabrera (2002). Instead, we used several open-ended questions to allow the interviewees to freely express their views. Each interview took at least two hours. An overview of the interviewees and their representativeness is provided in Table 3 below. The insights gained from these interviews also helped the authors to develop the study's hypotheses.

Among the interviewees, the online R\&D platform manager was responsible for knowledge management of the entire company and has been an advocate for the use of the online community to foster KS among R\&D staff. He was also one of the founders of the online R\&D platform and is knowledgeable about various aspects of online community design. He recalled that using HKFs was one of the original plans implemented to ensure a sufficient level of online interactions and KS in discussion forums. The two HKFs interviewed both felt that they had been making positive contributions to the online community by creating a more friendly and active atmosphere for regular members participating and sharing knowledge. Thus, it was a common understanding among HKFs that they were doing the company and online community members a favor without behaving illegally or unethically. We also interviewed a regular member of the online R\&D platform. He had been an active member of the online community since the platform's establishment. He fully understood the logic of HKFs and believed strongly that if HKFs were used appropriately, they might well serve a good purpose for the company and employees alike.

When asked how HKFs functioned or should function on the online R\&D platform, the interviewees provided interesting insights about the roles of HKFs. The main insights from the pre-study interviews are summarized below.

(1) HKFs' identities: There is no hierarchy among HKFs on the online communities, because they use an alias for their usernames and their real identities are not always known. An HKF is usually aware of the existence of other HKFs. An HKF may be active in multiple discussion forums under different topic categories and may also have multiple usernames, appearing with multiple identities without being identified as the same person.

(2) HKFs' motivations: Because the key performance indicators of KS for each of the company's R\&D institutes are summarized, evaluated, and reported by the manager of the online R\&D platform to top management, the HKFs at each institute have some incentive to keep intervening in online $\mathrm{R} \& \mathrm{D}$ communities. There is also a monthly monetary award, granted at the corporate level, for the best contributing community members. Therefore, expectations of personal advancement in terms of status, competence, promotion, or an increase in salary may directly motivate them to actively play their role as an HKF. HKFs constantly monitor and participate in the discussions on the online forums that match their interests. However, some HKFs also have intrinsic motivations: 
Table 3. An overview of interviewees and their background

\begin{tabular}{|c|c|c|c|}
\hline Functions & $\begin{array}{l}\text { Number of } \\
\text { persons } \\
\text { interviewed }\end{array}$ & Remarks & Commonly asked questions \\
\hline $\begin{array}{l}\text { Online R\&D } \\
\text { platform } \\
\text { manager }\end{array}$ & 1 & $\begin{array}{l}\text { This person oversees the entire } \\
\text { online R\&D platform. He has the } \\
\text { mandate to enhance the com- } \\
\text { pany's overall effectiveness of KS } \\
\text { and management. He also has } \\
\text { decision rights to implement new } \\
\text { approaches on the online R\&D } \\
\text { platform and he has access to } \\
\text { qualitative and quantitative } \\
\text { online log data. }\end{array}$ & $\begin{array}{l}\text { - Are you aware of the concept } \\
\text { of 'ghost' customers or players } \\
\text { in various online communi- } \\
\text { ties? Do you know if there are } \\
\text { similar roles in the company's } \\
\text { online R\&D platform? }\end{array}$ \\
\hline $\begin{array}{c}\text { Official } \\
\text { HKFs }\end{array}$ & 2 & $\begin{array}{l}\text { Two R\&D employees who have } \\
\text { the role of HKFs as part of their } \\
\text { work responsibilities. They have } \\
\text { hands-on experience of } \\
\text { "manipulating" the interactions } \\
\text { in the online R\&D communities. }\end{array}$ & $\begin{array}{l}\text { Do you think it is helpful to } \\
\text { use HKFs in the company's } \\
\text { online R\&D communities to } \\
\text { facilitate knowledge sharing? }\end{array}$ \\
\hline $\begin{array}{l}\text { Regular } \\
\text { active user }\end{array}$ & 1 & $\begin{array}{l}\text { An active user of the online } \mathrm{R} \& \mathrm{D} \\
\text { platform, thus a longtime } \\
\text { member of the communities. He } \\
\text { has insightful thoughts on how to } \\
\text { use HKFs to influence knowl- } \\
\text { edge interactions on online } \\
\text { communities in general, but he is } \\
\text { not fully aware that the company } \\
\text { actually is using HKFs. }\end{array}$ & $\begin{array}{l}\text { - What kinds of roles HKFs are } \\
\text { or should be playing? } \\
\text { - Are you aware of any HKFs in } \\
\text { the company's online R\&D } \\
\text { communities? }\end{array}$ \\
\hline
\end{tabular}

they find it psychologically rewarding to see other online community members learning from them and gain some benefit themselves from the experience.

(3) HKFs' interventions: HKFs recognize that finding a balance between overdoing it - resulting in overly heated online interactions and too powerful individual status - and underdoing it - resulting in ineffective user interactions in online communities - is an art unto itself. In other words, both the quantity and the quality of their online interventions matter, and a good balance between quantity and quality takes judgment and sensitivity. An HKF needs to be fairly knowledgeable in the topics where he/she intervenes. When an HKF continues contributing, he/she is more likely to be perceived as an expert who gradually becomes more and more influential in the online community.

\section{DATA AND METHODS}

\section{Data}

In designing the research study, we sought to both understand the effect of HKFs on KS outcomes within organizations and overcome the shortcomings of 
self-reporting common to the questionnaire method, the approach used in the majority of prior studies on KS effectiveness (e.g., Chen \& Hung, 2010; Haas \& Hansen, 2007; Lee, 2001; Ma \& Yuen, 2011). Thus, we chose to deploy a research design that fully explored the log data of an intrafirm online R\&D community for $\mathrm{KS}$, based on extensive content analysis of HKFs' objective online intervention behaviors, in order to reveal their actual effectiveness. We treated each online discussion thread as the unit of observation and conducted statistical analysis using different modeling techniques to examine the impact of quantity and quality of HKFs' online interventions on the KS outcomes in online R\&D communities. We observed the textual output of the company's online R\&D communities, giving a special focus on the quantity and quality of the HKFs' online interventions, during the two-month period of March-April, 2014 ${ }^{[2]}$, during which more than 50 percent of the R\&D personnel on average visited the online platform on a monthly basis. Discussion topics in different forums received different levels of attention, ranging from fewer than five visits to more than 300 visits and taking from less than one minute to more than three hours of viewing time.

Senior management of the company provided us with the names of all 23 HKFs (with their names, employee IDs, online user names, and email addresses) who were operating on the intrafirm R\&D communities during the study period. The online R\&D community manager granted us direct access to the online $\log$ data. Of the various sections ${ }^{[3]}$ of the online platforms, we focused on online Q\&A forums for two reasons: (1) they are the most active areas of the online R\&D platform; and (2) the discussions mostly form 'vertical questions', which assume that a correct answer exists and can be found, and 'horizontal questions', which invite negotiation around a plausible answer (Fahy, 2003). These types of questions and communication patterns match our research purpose.

The web log data of all the online threads in the Q\&A forums were used to calculate the quantity of HKF interventions and the length of each thread. To assess the content of online communications, we followed a 'netnographic' approach, as suggested by prior studies (Jeppesen \& Fredriksen, 2006). Netnography is an interpretive methodology, focusing on the textual output of Internet-related fieldwork (Kozinets, 1998). The 'netnographic' approach enables us to fully understand the context of a particular act of an HKF in a given situation without any potential misinterpretation. This serves as a solid foundation on which content analysis can subsequently be conducted to measure the variables that need qualitative judgment and evaluation, such as quality of intervention, solution found, and convergent and divergent discussion.

\section{Variables and Measures}

First, quantitative approaches to measuring the amount of online interaction are well known, and it is relatively straightforward to measure the number of posts in each thread (Masters \& Oberprieler, 2004; Nisbet 2004). Therefore, one

(C) 2018 The International Association for Chinese Management Research 
independent variable, quantity of $H K F$ intervention, is measured by counting the number of HKFs' posts (this is possible because we know the user IDs of all $\mathrm{HKFs}$. However, we are also interested in a related but different measure of $\mathrm{HKF}$ intervention, measured by the number of unique HKFs involved in each thread. These two measures for quantity of HKFs' interventions are highly correlated (and thus not to be included in the same regression models as independent variables) but represent different aspects of the quantity variable. In the analysis, we run regressions for these two measures of quantity of HKFs' intervention separately.

Second, regarding the electronic format of KS with regard to content and process, there are several ways of measuring quality of online intervention discussed in the literature, which primarily recommends that researchers ask other community members or managers to rate the online contributions of a particular type of user (Haas \& Hansen, 2007). This approach requires raters to have a good understanding of the context of the contributions to be rated in order to ensure that the rating is impartial and reliable. However, we could not use such an approach because the practice of using HKFs amounted to a corporate secret - only a handful of managers knew about it. If we had followed the rating approach by involving other managers and employees, it would have inevitably revealed the identities of the HKFs. Therefore, we had to use a different approach.

In this study, we follow Haas and Hansen (2007) and focus on the concept of level of rework. Level of rework measures the degree to which a user's online contribution requires additional work by other online users before it becomes sufficiently informative and useful. Level of rework in this sense is conceptually representative of the concept of intervention quality, especially as our observation focuses on the online Q\&A forums, where helpful information and useful solutions are expected among users. An online intervention of high quality should have a low level of rework. Thus, quality of an HKF's online intervention is a reversed measure of level of rework. We use content analysis (Wickersham \& Dooley, 2006) to measure quality of intervention, using the following coding convention: $0=$ no useful information; 1 = suggesting a perspective, viewpoint, or potential way of seeking solutions; 2 = referring to a concrete document, link, media file, or authoritative viewpoint with evidence, but requires further learning by those who raised the question; 3 = providing direct answers to the question with certainty. If there is more than one intervention by an $\mathrm{HKF}$, we take the average value of the quality measure.

Three dependent variables were identified. The first, length of discussion, is measured by counting the number of total posts in each thread and subtracting the number of posts made by HKFs.

Next, whether a solution has been found in each discussion thread is measured by a binary variable, which draws on straightforward information from each discussion thread. In some typical cases, solutions feature a clear statement by the question raiser, saying, for instance, 'Aha, problem solved, thank you guys so much!', 
'This works! Awesome?', or 'I have just tested (it), XXX was so helpful!' Many other cases became an open discussion without any concluding remarks or testimony of effectiveness from anyone in the discussion thread.

Third, there has been a long tradition of measuring divergent and convergent thinking at an individual level, using the so-called 'alternative uses task' (AUT) test and 'remote associates task' (RAT) test, respectively (Hommel, Colzato, Fischer, \& Christoffels, 2011; Jauk, Benedek, \& Neubauer, 2012). These tests have been criticized for their potential bias, derived from subjective evaluation of quality scoring (Runco \& Acar, 2012). These traditional tests do not suit our research purpose, because the focus of our unit of observation is convergent and divergent patterns of discussion occurring collectively in online discussion threads. Therefore, relying on content analysis of original text and context, we used the following coding convention to judge the occurrence of divergent thinking: (1) different opinions, which are complementary to each other, appear; (2) different opinions, which disagree with one another, appear; (3) different opinions, which seem completely unrelated, appear. When any of these three scenarios takes place, we code the variable as ' 1 ', otherwise ' 0 '. Based on the same approach, we use the following coding convention to judge the occurrence of convergent thinking: (1) all following posts converge eventually, agreeing on a plausible or convincing solution to the original question; (2) the question raiser and the following posts that attempt to answer the question converge eventually, but the question does not necessarily find an answer. It could be the case that the question raiser confirmed that the suggestion or further inquiries from the posts are relevant and important to the original question. This could also happen when a question was not clearly defined or vague, or lacked visual information, so that a few more rounds of communication were needed to clarify the question and related issues. This process itself is a good learning outcome; (3) the question raiser and the following posts converge by recognizing the relevance and importance of the same question, and other members in the community share an interest in getting the answer to the same question as well. When any of these scenarios takes place, we code the variable as ' 1 ', otherwise ' 0 '. Among the 379 observations, $14.8 \%$ of the cases are convergent ONLY, $30.9 \%$ are divergent ONLY, and only $1.6 \%$ are BOTH convergent and divergent.

When using content analysis, a coding team of two of the co-authors and two research assistants with both engineering and management backgrounds independently coded the relevant variables. Then we compared our coding results to identify and discuss any inconsistencies and agree on final scores. A list of coding schemes of the variables based on content analysis of online communications are provided in Table 4, with a number of real examples from online discussion threads.

Finally, we also include two dummies as control variables: (1) whether a discussion thread was initiated by an HKF; and (2) whether the discussion thread took place in March versus April, 2014, to control for any unobservable variance pertaining to time. 
HKF1: who knows how to get the corporation standard yellow logo color in $\mathrm{PRO} / \mathrm{E}$ ?

HKF2: Go to 'View-system color arrange- set to original'

HKF1: No, what I was asking was how to manually set the standard yellow logo color of our corporation based on the tune balance among red yellow and blue base colors?

User1: You cannot get such a tune balance ratio. It was done by advanced color tuning technology and it did not have a standard value. It will be extremely hard to manually fine tune it in PRO/E program by adjusting red, yellow and blue base colors.
User1: I have two questions: first, who knows why InteCAD 2004 cannot be linked to Windchill system anymore after installing Windows 7? The moment I link it to Windchill, then endless waiting time, like the system crashed.; Second, the PRO/E program became useless after installing Windows 7, as I cannot detect leaking in the interactive assessment model. It was not like this before. Who knows anything about these?

HKF1: Unless it is A version unpublished status, otherwise it must be done by admin right to rename. Please check the following link (a URL provided to a technical instruction document)

HKF1: Please try to use Modelcheck to do model quality check (a URL provided to a technical instruction document)

User2: I have experienced the first situation as well. I think I forgot to check 'system compatibility'. You just need to uninstall InteCAD and AutoCAD and then reinstall them again.

HKF1: (provided a URL for downloading a file to install AutoCAD-IntelCAD and PDM HKF1: Is it possible that IntelCAD was not properly installed? Maybe you can reinstall it. User2: I just did it as you suggested, but these problems appeared 2 days after the reinstallation again. :)

User3: Try this '^^^ USER FATAL MESSAGE 9050 (SEKRRS) \r \n ^^^ RUN TERMINATED DUE TO EXCESSIVE PIVOT RATIOS IN MATRIX KLL. $\backslash \mathrm{r} \backslash \mathrm{n} \wedge$ ^^ USER ACTION: CONSTRAIN MECHANISMS WITH SPCI OR SUPORTI

ENTRIES OR $\backslash r \backslash n$ SPECIFY PARAM,BAILOUT,-1 TO \r $\backslash n$ CONTINUE THE RUN WITH MECHANISMS. $\backslash r \backslash n \backslash r \backslash n$ NASTRAN should give a warning message saying that IN = "TWO" is not $\backslash \mathrm{r} \backslash \mathrm{n}$ allowed and that the suggested IN = "THREE" is used instead,

and the run $\backslash r \backslash n$ should continue. $\backslash r \backslash n \backslash r \backslash n$ Avoidance: Use IN equal to "THREE".

User1: Wow, awesome!

HKF2: Here is another way! (provided a URL of a webpage on how to solve IntelCAD and Winchill problems)

User2: Thanks a lot! The commands in Windchill's menu are working now, but the tools in tables disappeared.

HKF3: We need to share more knowledge here. 


\begin{tabular}{|c|c|c|c|}
\hline & & Discussion thread example 1 & Discussion thread example 2 \\
\hline Variables & Coding schemes & Goding & Coding \\
\hline $\begin{array}{l}\text { Quality of } \\
\text { intervention }\end{array}$ & $\begin{array}{l}0=\text { no useful information } \\
1=\text { suggesting a perspective, a viewpoint, } \\
\text { or a potential way of seeking solutions } \\
2=\text { referring to a concrete document, } \\
\text { link, media file, or authoritative view- } \\
\text { point with evidence, but requires further } \\
\text { learning by the question raisers } \\
3=\text { providing direct answers to the } \\
\text { question with certainty }\end{array}$ & $\begin{array}{l}\text { Quality of intervention }=(1+3+1) / 3= \\
1.667\end{array}$ & Quality of intervention $=(2+2+2+1+2+0) / 6=1.5$ \\
\hline Solution found & $\begin{array}{l}\text { An answer to the question is found within } \\
\text { a discussion thread "l", otherwise "0" }\end{array}$ & Coded as ' 1 ' & Coded as ' 0 ' \\
\hline $\begin{array}{l}\text { Convergent } \\
\text { discussion }\end{array}$ & $\begin{array}{l}\text { If any of the following happened, coded } \\
\text { as ' } 1 \text { ', otherwise ' } 0 \text { ': (1) all following } \\
\text { posts converge eventually, agreeing on a } \\
\text { plausible or convincing solution to the } \\
\text { original question; (2) the question raiser } \\
\text { and the following posts that attempted } \\
\text { to answer the question converge even- } \\
\text { tually, but the question does not neces- } \\
\text { sarily find an answer. (3) The question } \\
\text { raiser and the following posts converge } \\
\text { by recognizing the relevance and } \\
\text { importance of the same question, and } \\
\text { other members in the community share } \\
\text { the interest in getting the answer to the } \\
\text { same question as well. }\end{array}$ & Coded as ' 1 ' & Coded as ' 1 ' \\
\hline
\end{tabular}


Table 4. Continued

\begin{tabular}{|c|c|c|c|}
\hline & & Discussion thread example 1 & Discussion thread example 2 \\
\hline $\begin{array}{l}\text { Divergent } \\
\text { discussion }\end{array}$ & $\begin{array}{l}\text { If any of the following happened, coded } \\
\text { as ' } 1 \text { ', otherwise ' } 0 \text { ': (1) different opi- } \\
\text { nions, which are complementary to } \\
\text { each other, appear; (2) different opi- } \\
\text { nions, which disagree with one another, } \\
\text { appear; (3) different opinions, which } \\
\text { seem completely unrelated, appear. }\end{array}$ & Coded as '0' & Coded as ' 0 ' \\
\hline
\end{tabular}




\section{Statistical Models}

In this study, the unit of observation for data analysis is each discussion thread, instead of each individual or the entire online community. As the natures of the dependent variables for our hypotheses are quite different, we use different statistical modeling techniques for each. The dependent variable for $\mathrm{H} 1, \mathrm{H} 2$, and $\mathrm{H} 3$ is the length of discussion, a count variable. Thus, we use a Poisson regression model to appropriately count so that discrete events can be modeled (Cameron \& Trivedi, 2005). To test H4-H12, we use binary logistic regressions because the dependent variables for these hypotheses are binary variables.

\section{RESULTS}

During March and April of 2014 there were 379 online discussion threads and 1,717 posts in total in the R\&D Q\&A forums; thus the average length of a thread is 4.53 posts. On average, HKFs made 1.32 posts per thread. Interestingly, most of the threads were not initiated by an HKF (mean value = 0.17). In Table 5, the mean and standard deviation of all variables are presented with their correlations. Among all the independent variables and control variables, we found that several correlations are moderately high, for instance, between $H K F$ s initiation and the number of unique HKFs (0.508), between HFKs initiation and the number of HKFs' posts (0.533), and between quality of intervention and the number of HKF' ' posts (0.554). We further checked the variance inflation factors (VIF) for all the independent and control variables against the corresponding dependent variables: we found that no variable has a VIF value higher than 2, much lower than the critical value, 10. Thus, multicollinearity is not a concern. Furthermore, as the two measures of quantity of HKFs' interventions (the number of unique HKFs in each thread and the number of HKFs' posts) are not included in the same estimation models in any case, the high correlation between these two measures $(0.737)$ is not of concern.

Table 6 presents the results of the Poisson regression to test $\mathrm{H} 1, \mathrm{H} 2$, and $\mathrm{H} 3$, which predict the impact of quantity and quality of HKFs' online intervention on the length of discussion as the dependent variable. We first introduce a base model (model 0), using only two main control variables: HKF's initiation and month. Then, we introduce the two main effect variables, quantity and quality of online intervention (models 1 and 3). Next, we introduce the interaction terms of quantity and quality of HKFs intervention into the model (models 2 and 4). The Wald Chi ${ }^{2}$ statistics for all the models are significant compared to an intercept-only model. In models 1 and 2, the quantity of intervention is measured by the number of unique HKFs, while in models 3 and 4 it is measured by the total number of HKFs' posts.

We find that the quantity of intervention has a positive and significant effect on the dependent variable in both models 1 and $2(\beta=0.354, p<0.01 ; \beta=0.514, p<$ 0.01 , respectively). When it is measured differently, the quantity of intervention has

(C) 2018 The International Association for Chinese Management Research 
Table 5. Descriptive statistics and correlations $(\mathcal{N}=379)$

\begin{tabular}{|c|c|c|c|c|c|c|c|c|c|c|c|}
\hline Variables & Mean & s. $d$. & 1 & 2 & 3 & 4 & 5 & 6 & 7 & 8 & 8 \\
\hline 1. HKFs initiation & 0.17 & 0.373 & 1 & & & & & & & & \\
\hline 2. Month (March 2014) & 0.17 & 0.375 & 0.045 & 1 & & & & & & & \\
\hline 3. Number of unique HKFs & 0.91 & 1.030 & $0.508 * *$ & -0.057 & 1 & & & & & & \\
\hline 4. Number of HKFs' posts & 1.32 & 2.022 & $0.533^{* *}$ & 0.001 & $0.737 * *$ & 1 & & & & & \\
\hline 5. Quality of intervention & 0.79 & 0.939 & $0.170^{* * *}$ & 0.037 & $0.554 * *$ & $0.385^{* * *}$ & 1 & & & & \\
\hline 6. Length of discussion & 3.21 & 3.067 & $-0.130^{*}$ & -0.095 & $0.240 * *$ & $0.230 * *$ & $0.108 *$ & 1 & & & \\
\hline 7. Solution found & 0.47 & 0.500 & $0.122^{*}$ & -0.097 & $0.359 * *$ & $0.241^{* *}$ & $0.359 * *$ & $0.231 * *$ & 1 & & \\
\hline 8. Convergent discussion & 0.32 & 0.469 & 0.099 & -0.072 & $0.258 * *$ & $0.157 * *$ & 0.082 & $0.326^{* *}$ & $0.142^{* * *}$ & 1 & \\
\hline 9. Divergent discussion & 0.16 & 0.370 & -0.006 & -0.009 & $0.219 * *$ & $0.226^{* * *}$ & $0.138 * *$ & $0.339 * *$ & 0.058 & $-0.215^{* *}$ & 1 \\
\hline
\end{tabular}

Note: Significance levels: $* \mathrm{p}<0.05 ; * * \mathrm{p}<0.01$ 
Table 6. Results of Poisson Regression Models for H1, H2, and H3

\begin{tabular}{|c|c|c|c|c|c|}
\hline \multirow[b]{3}{*}{ Intercept } & \multirow{2}{*}{ Model 0} & Model 1 & Model 2 & Model 3 & Model 4 \\
\hline & & \multicolumn{2}{|c|}{$\begin{array}{l}\text { (Quantity of intervention measured } \\
\text { by number of unique HKFs) }\end{array}$} & \multicolumn{2}{|c|}{$\begin{array}{l}\text { (Quantity of intervention measured } \\
\text { by number of HKFs' posts) }\end{array}$} \\
\hline \multirow{2}{*}{\multicolumn{6}{|c|}{ Control variables }} \\
\hline & & & & & \\
\hline HKFs initiation & $-0.369(0.088) * * *$ & $-0.863(0.099)^{* * * *}$ & $-0.892(0.099)^{* * * *}$ & $-0.930(0.110)^{* * * *}$ & $-1.087(0.1170) * * *$ \\
\hline Month (March 2014) & $-0.250(0.084)^{* * *}$ & $-0.155(0.085)^{*}$ & $-0.119(0.085)$ & $-0.314(0.086) * * *$ & $-0.211(0.086)^{* *}$ \\
\hline \multicolumn{6}{|l|}{ Main effects } \\
\hline Quantity of intervention & & $0.354(0.033)^{* * * *}$ & $0.514(0.052) * * *$ & $0.138(0.012) * * *$ & $0.271(0.026) * * *$ \\
\hline Quality of Intervention & & $-0.063(0.037)^{*}$ & $0.094(0.0543)^{*}$ & $0.035(0.031)$ & $0.123(0.036) * * *$ \\
\hline \multicolumn{6}{|l|}{ Interaction effect } \\
\hline Quantity of intervention * Quality of intervention & & & $-0.170(0.045)^{* * * *}$ & & $-0.098(0.018) * * *$ \\
\hline \multicolumn{6}{|l|}{ Model statistics } \\
\hline Wald $C h i^{2}$ & $29.761^{* * *}$ & $154.230 * * *$ & $169.680 * * *$ & $146.999 * * *$ & $174.937 * * *$ \\
\hline df (compared against intercept-only model) & 2 & 4 & 5 & 4 & 5 \\
\hline
\end{tabular}

Notes: Significance levels: * $\mathrm{p}<0.10 ; * * \mathrm{p}<0.05 ; * * \mathrm{p}<0.01$; Standard errors in brackets; $\mathcal{N}=379$ 

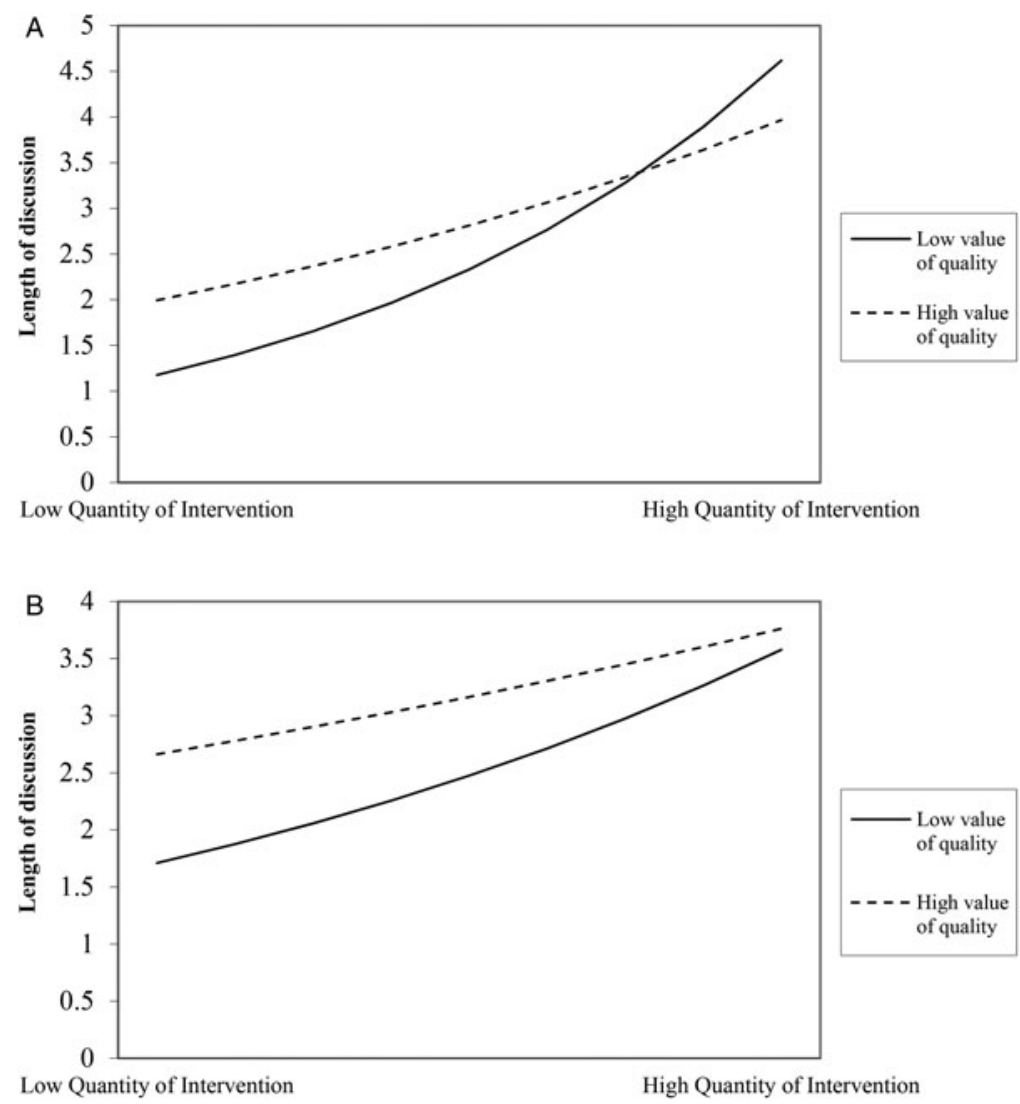

Figure 1A-1B. Interaction effect of quantity, moderated by quality, of HKFs intervention on length of discussion $(\mathrm{H} 3)^{*}$

Notes: *Low value of moderator is defined as one s.d. below mean, and high value is defined as one s.d. above mean. Value of moderator is centered.

a positive and significant effect on the dependent variable in both models 3 and 4 as well $(\beta=0.138, p<0.01 ; \beta=0.271, p<0.01$, respectively). Therefore, $\mathrm{H} 1$ is supported. However, the effect of $H K F s^{\prime}$ intervention quality has a marginal effect on the dependent variable in models 1 and $2(\beta=-0.063, p<0.10 ; \beta=0.094, p<$ 0.10 , respectively). Only when the interaction term of quantity and quality of $H K F s^{\prime}$ intervention is introduced in model 4 , does the quality of intervention show a positive and significant effect $(\beta=0.123, p<0.01)$. Therefore, $\mathrm{H} 2$ is only partially supported. H3 predicts a negative moderating effect of quality on the positive effect of quantity. We find that the coefficients of quantity times quality are both negative and significant in models 2 and $4(\beta=-0.170, p<0.01 ; \beta=-0.098, p<0.01$, respectively). Thus, H3 seems supported. The interaction effect is plotted and shown in Figure 1A and 1B. However, by calculating the standardized mean difference (SMD) effect size for the moderating effect, we find that SMD is -0.25 (with an estimation of $[-0.364,-0.129]$ at the $95 \%$ confidence interval) when quantity of intervention is measured by number of unique HKFs. The SMD effect size for the 

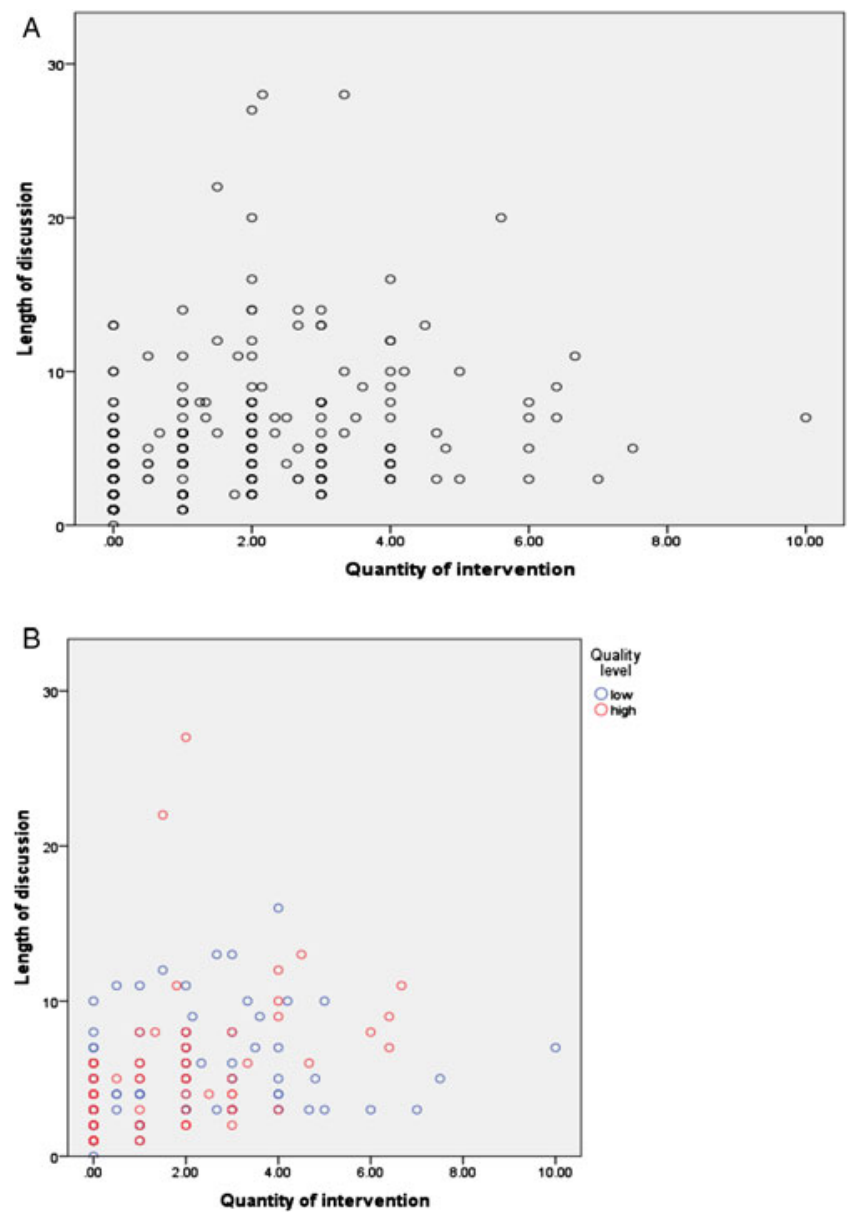

Figure 2A-2B. Scatterplot for effect of quantity, moderated by quality, of HKFs intervention on length of discussion (H3) (Quantity of intervention measured by number of unique HKFs)

moderating effect is -0.156 (with an estimation of $[-0.209,-0.10]$ at the $95 \%$ confidence interval) when quantity of intervention is measured by number of HKFs' post. These tests about effect size indicate no effect.

The actual effect of quantity of intervention, given the levels of quality of intervention, also can be intuitively interpreted by visualizing the actual data. Following the recent call for paying more attention to making sense of actual data in management research beyond showing coefficient estimates, standard errors, and significance levels (Greve, 2018; Levine, 2018), we make scatterplots to further inspect the hypothesized moderating effect. Figure $2 \mathrm{~A}$ shows all cases with value of length of discussion vis-à-vis quantity of intervention, which is measured by the number of unique HKFs. Figure 2B shows cases with low or high levels of quality of intervention separately. Similarly, Figure 3A shows the relationship between length of discussion and quantity of intervention, which is measured by the number of HKFs' posts. Figure 3B separates cases with low or high levels of quality of intervention. We find that regardless in which way the quantity of 

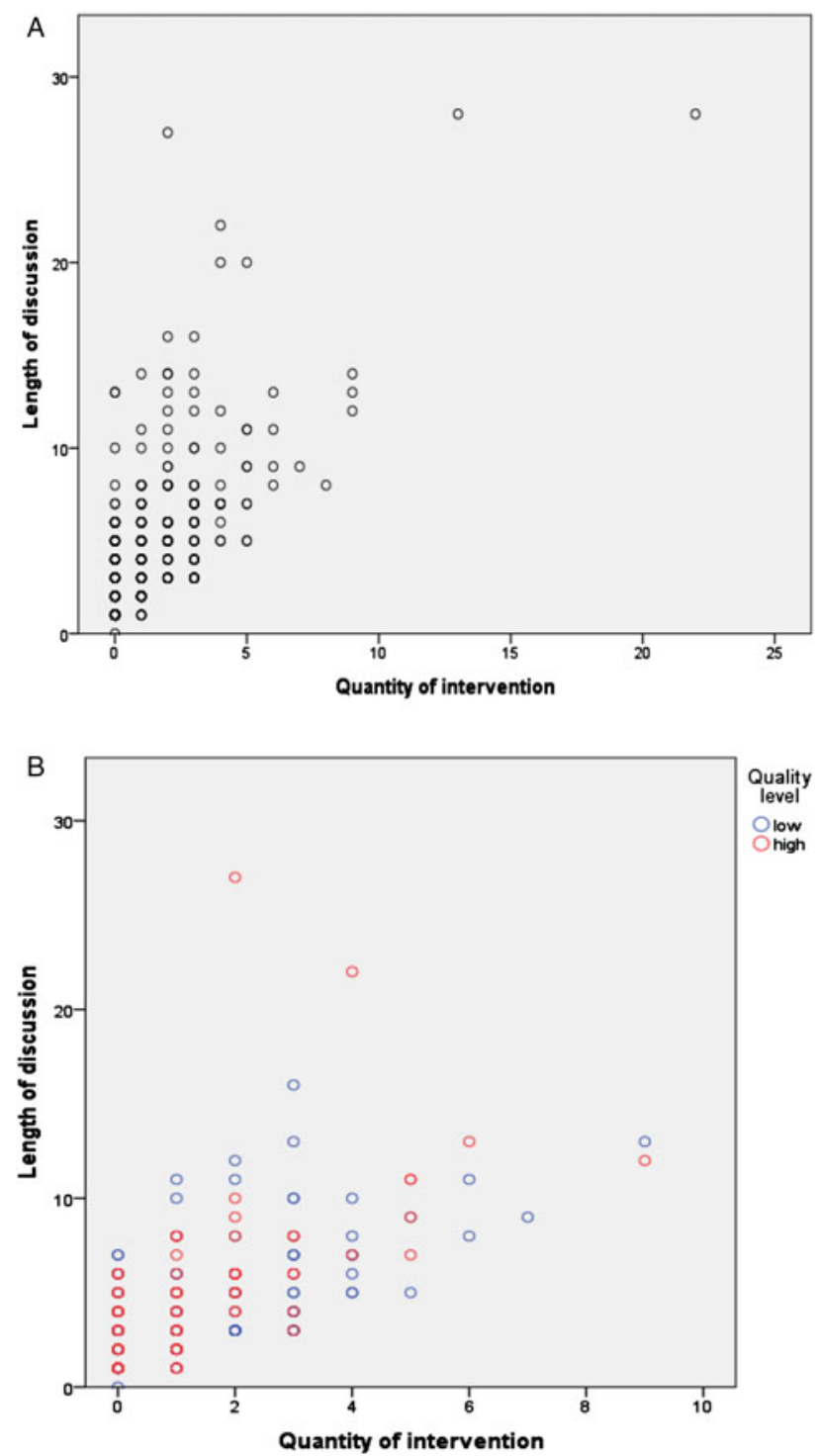

Figure 3A-3B. Scatterplot for effect of quantity, moderated by quality, of HKFs intervention on length of discussion (H3) (Quantity of intervention measured by number of HKFs' posts)

intervention is measured, it is not easy to visually judge that a seemingly positive relationship between invention quantity and length of discussion is less evident for cases with high levels of quality of intervention than those with low levels of quality of intervention. Thus, it makes sense to conclude that H3 is not supported.

To test H4, H5, and H6, which assess the impact of HKFs' intervention quantity and quality on the solution found, we ran a binary logistic regression because the dependent variables with a binary value; the results are shown in Table 7 . Both models meet the Hosmer-Lemeshow test (non-significant chi-square), indicating that the data fit the models well. In the first model, the quantity of intervention 
Table 7. Results of Binary Logistic Regression Models for H4, H5, and H6

\begin{tabular}{|c|c|c|}
\hline Dependent variables & $\begin{array}{l}\text { Solution found } \\
\text { (Quantity of intervention } \\
\text { measured by number } \\
\text { of unique HKFs) }\end{array}$ & $\begin{array}{l}\text { Solution found } \\
\text { (Quantity of intervention } \\
\text { measured by number } \\
\text { of HKFs' posts) }\end{array}$ \\
\hline \multicolumn{3}{|l|}{ Independent variables } \\
\hline Constant & $-0.947(0.177)^{* * *}$ & $-0.932(0.172)^{* * *}$ \\
\hline \multicolumn{3}{|l|}{ Control variables } \\
\hline HKFs initiation & $-0.246(0.349)$ & $-0.126(0.515)$ \\
\hline Month (March 2014) & $-0.585(0.321)^{*}$ & $0.580(0.318)^{*}$ \\
\hline \multicolumn{3}{|l|}{ Main effects } \\
\hline Quantity of intervention & $\begin{array}{c}0.619(0.238)^{* * *} \\
(\text { odds ratio }=1.858)\end{array}$ & $\begin{array}{c}0.470(0.159)^{* * * *} \\
(\text { odds ratio }=1.600)\end{array}$ \\
\hline Quality of intervention & $\begin{array}{c}0.616(0.231)^{* * *} \\
(\text { odds ratio }=1.852)\end{array}$ & $\begin{array}{c}1.007(0.168)^{* * *} \\
(\text { odds ratio }=2.737)\end{array}$ \\
\hline \multicolumn{3}{|l|}{ Interaction effect } \\
\hline Quantity*Quality of intervention & $-0.088(0.203)$ & $\begin{array}{c}-0.286(0.108) * * * \\
(\text { odds ratio }=0.752)\end{array}$ \\
\hline \multicolumn{3}{|l|}{ Model statistics } \\
\hline Cox \& Snell R ${ }^{2}$ & 0.170 & 0.165 \\
\hline Hosmer and Lemeshow Test (df) & $4.545(6)$ & $5.358(6)$ \\
\hline
\end{tabular}

Notes: Significance levels: $* \mathrm{p}<0.10 ; * * \mathrm{p}<0.05 ; * * * \mathrm{p}<0.01 ;$ Standard errors in brackets; $\mathcal{N}=379$

is measured by the number of unique HKFs, while in the second model it is measured by the total number of HKFs' posts. H4 predicts that the quantity of HKFs' online interventions is positively associated with the likelihood that solutions will be found. The results show that the quantity of intervention has a positive and significant effect in both models $(\beta=0.619, p<0.01$, odds ratio $=1.858 ; \beta=0.470, p<$ 0.01 , odds ratio $=1.600$, respectively), supporting H4. Next, H5 predicts that the quality of HKFs' intervention is positively associated with the likelihood that solutions will be found. We find that the quality of intervention has a positive and significant effect in both models $\beta=0.616, p<0.01$, odds ratio $=1.852$; $\beta=1.007, p<0.01$, odds ratio $=2.737$, respectively), supporting H5. When the interaction term of quantity and quality is introduced into the models, we find that it shows a negative and significant effect only in the second model, where the quantity is measured by the number of HKFs' posts $(\beta=-0.286, p<0.05$, odds ratio = $0.752)$, but not in the first model. Thus, H6 is only supported only when intervention quantity is specifically measured by the number of HKF's posts with a relatively small effect size of the moderating effect of 0.752 (odds ratio). The interaction effect is plotted and shown in Figure 4. We also make scatterplots to further inspect the hypothesized moderating effect. Figure $5 \mathrm{~A}$ shows all cases regarding the relationship between predicted probability of solution found and quantity of intervention, which is measured by the number of HKFs' posts.

(C) 2018 The International Association for Chinese Management Research 


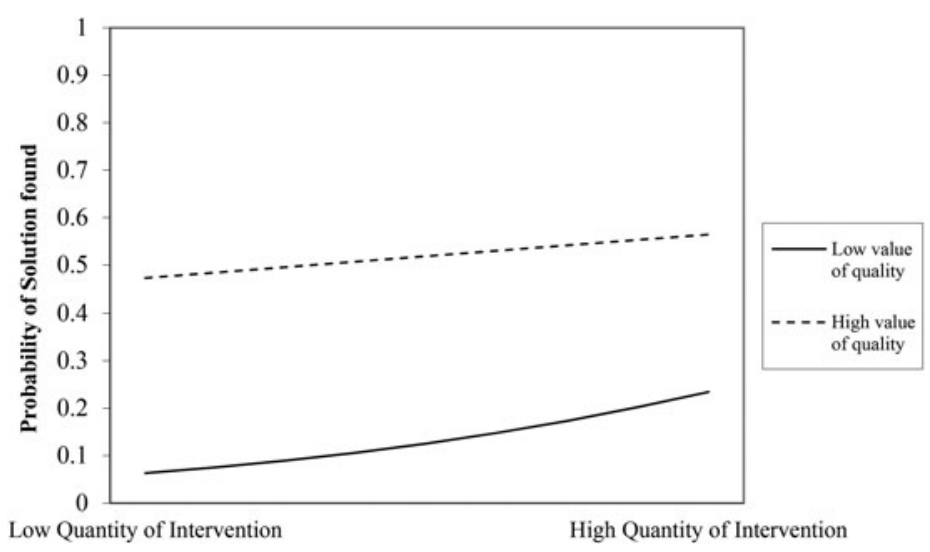

Figure 4. Interaction effect of quantity, moderated by quality, of HKFs intervention on solution found (H6)* (quantity of intervention measured by number of HKFs' posts)

Notes: *Low value of moderator is defined as one s.d. below mean, and high value is defined as one s.d. above mean. Value of moderator is centered.

Figure 5B shows cases with low and high levels of quality of intervention separately. It is visually not difficult to figure out that, the cases with a low level of quality of intervention align with a positive effect line in a better shape than those with a high level of quality of intervention.

To test H7, H8, and H9, which assess the impact of HKFs' intervention quantity and quality on convergent discussion, binary logistic regression is used again; the results are shown in Table 8 . In the first model, the quantity of intervention is measured by the number of unique HKFs, while in the second model it is measured by the total number of HKFs' posts. The first model does not meet the HosmerLemeshow test, indicating that the model is not a good fit. Therefore, we only count the results shown in the second model, where quantity of intervention is measured by the number of HKFs' posts. $\mathrm{H} 7$ predicts that the quantity of HKFs' online interventions is positively associated with convergent thinking. The results show that the quantity of intervention has a positive and significant effect $(\beta=0.336, p$ $<0.05$, odds ratio $=1.400)$, supporting H7. Next, H8 predicts that the quality of HKFs' interventions is positively associated with convergent thinking. We find that the coefficient of quality of intervention is positive but not significant. Thus, H8 is not supported. This is probably because convergent thinking in a group level actually takes place offline after high quality posts are viewed by employees, and we have no means to observe offline convergent thinking. When the interaction term of quantity and quality is introduced into the model, we find that it shows a negative and significant effect with a marginal level of significance $\beta=$ $-0.170, p<0.10$, odds ratio $=0.8442$ ). Thus, H9 is only marginally supported when intervention quantity is measured by the number of HKFs' posts with a small effect size of the moderating effect of 0.8842 (odds ratio). The interaction effect is plotted and shown in Figure 6. We also make scatterplots to visually inspect the hypothesized moderating effect. Figure 7A shows all cases regarding 

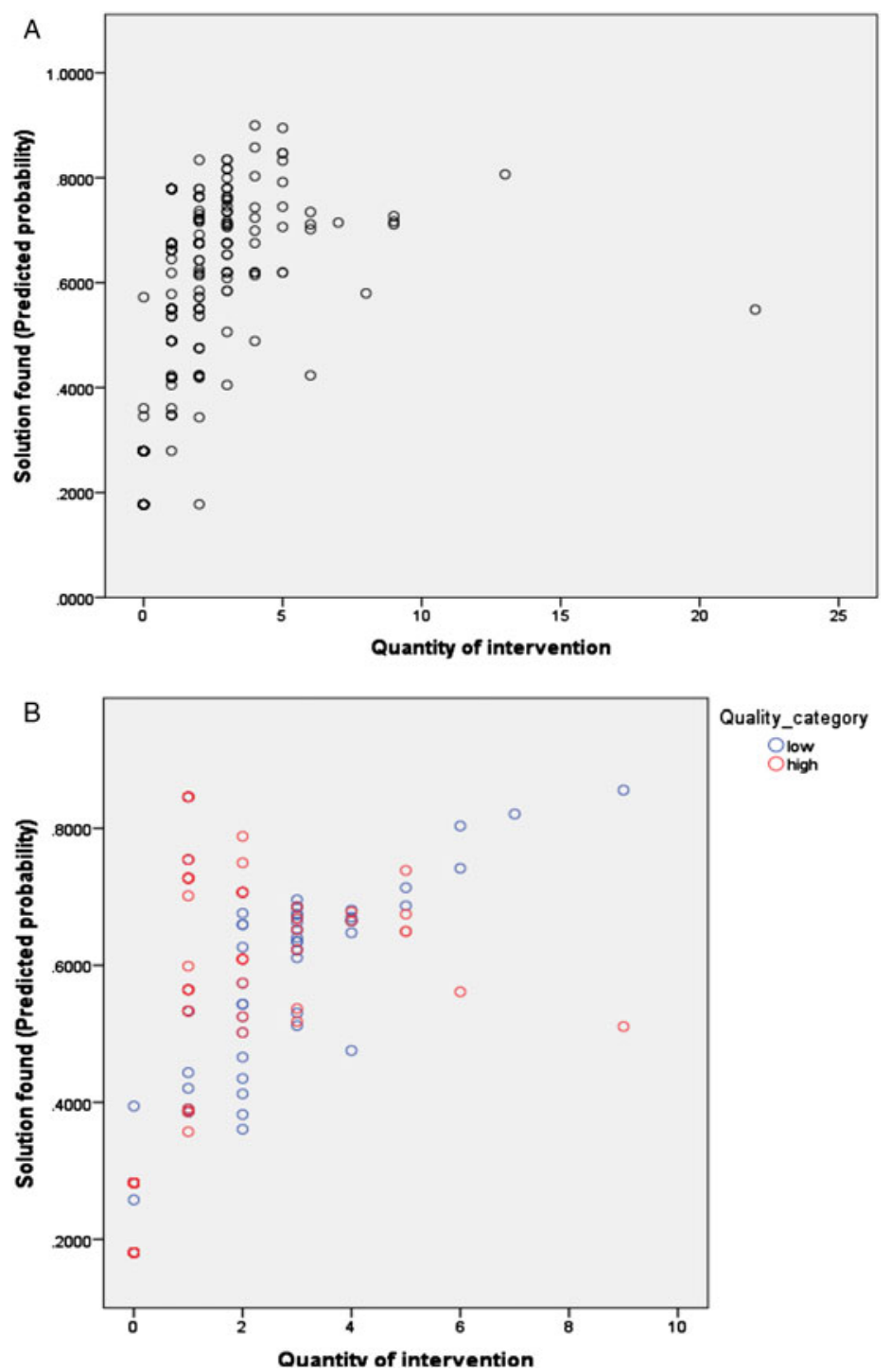

Figure 5A-5B. Scatterplot for effect of quantity, moderated by quality, of HKFs intervention on solution found (H6) (Quantity of intervention measured by number of HKFs' posts)

the relationship between predicted probability of convergent discussion and quantity of intervention, which is measured by the number of HKFs' posts. Figure $7 \mathrm{~B}$ shows cases with low and high levels of quality of intervention separately. It is visually not difficult to figure out that the cases with a low level of quality of intervention align with a positive effect line in a better shape than those with a high level of quality of intervention.

To test H10, H11, and $\mathrm{H} 12$ regarding the impact of HKFs' intervention quantity and quality on divergent discussion, we use binary logistic regression as well; the results are shown in Table 9. In the first two models, the quantity of intervention is measured by the number of unique HKFs, while in the last two models it is 
Table 8. Results of Binary Logistic Regression Models for H7, H8, and H9

\begin{tabular}{|c|c|c|}
\hline Dependent variables & $\begin{array}{l}\text { Convergent discussion } \\
\text { (Quantity of intervention } \\
\text { measured by number } \\
\text { of unique HKFs) }\end{array}$ & $\begin{array}{l}\text { Convergent discussion } \\
\text { (Quantity of intervention } \\
\text { measured by number of } \\
\text { HKFs' posts) }\end{array}$ \\
\hline \multicolumn{3}{|l|}{ Independent variables } \\
\hline Constant & $-1.159(0.185)^{* * *}$ & $-1.026(0.173)^{* * *}$ \\
\hline \multicolumn{3}{|l|}{ Control variables } \\
\hline HKFs initiation & $-0.288(0.347)$ & $-0.036(0.350)$ \\
\hline Month (March 2014) & $-0.296(0.326)$ & $-0.379(0.321)$ \\
\hline \multicolumn{3}{|l|}{ Main effects } \\
\hline Quantity of intervention & $\begin{array}{c}0.751(0.229) * * * \\
(\text { odds ratio }=2.120)\end{array}$ & $\begin{array}{c}0.336(0.145)^{* *} \\
(\text { odds ratio }=1.400)\end{array}$ \\
\hline Quality of intervention & $-0.107(0.224)$ & $0.235(0.157)$ \\
\hline \multicolumn{3}{|l|}{ Interaction effect } \\
\hline Quantity*Quality of intervention & $-0.093(0.185)$ & $\begin{array}{c}-0.170(0.102)^{*} \\
(\text { odds ratio }=0.844)\end{array}$ \\
\hline \multicolumn{3}{|l|}{ Model statistics } \\
\hline Cox \& Snell $\mathrm{R}^{2}$ & 0.072 & 0.037 \\
\hline Hosmer and Lemeshow Test (df) & 10.716 (6) Not fitting well & $7.356(6)$ \\
\hline
\end{tabular}

Notes: Significance levels: $* \mathrm{p}<0.10 ; * * \mathrm{p}<0.05 ; * * * \mathrm{p}<0.01$; Standard errors in brackets; $\mathcal{N}=379$

measured by the total number of HKFs' posts. All models meet the Hosmer-Lemeshow test, indicating that the data fit the models well. In the first model, we include quantity and quality of intervention and the squared term of quality of intervention. In the second model, we add the interaction term between the quantity of intervention and the squared term of quality of intervention. We use the same steps for the last two models. $\mathrm{H} 10$ predicts that the quantity of HKFs' online interventions is positively associated with divergent thinking. The results show that the quantity of intervention has a positive and significant effect in all models for both measures of quantity of intervention $(\beta=0.532, p<0.01$, odds ratio $=1.702 ; \beta=0.642, p<0.01$, odds ratio $=1.899 ; \beta=0.306, p<0.01$, odds ratio $=1.358 ; \beta=0.335, p<0.01$, odds ratio $=1.427$, respectively). Thus, $\mathrm{H} 10$ is supported. Next, H11 predicts that the quality of HKFs' interventions has an inverted U-shaped relationship with divergent thinking. We find that the coefficient of the square term of quality of intervention is negative and marginally significant only in the third model, where quantity of intervention is measured by the number of HKFs' posts $(\beta=-0.326, p<0.10)$. Thus, H11 only finds weak support. Finally, H12 predicts that the inverted U-shaped relationship between the quality of HKFs' interventions and divergent discussion is more evident when the quantity of interventions is large. However, the results in Table 9 for both types of measures for quantity of intervention show no significant effect. Thus, H12 is not supported. This weak result for $\mathrm{H} 11$ and non-support for $\mathrm{H} 12$ can be interpreted that quality of intervention alone does not matter much 


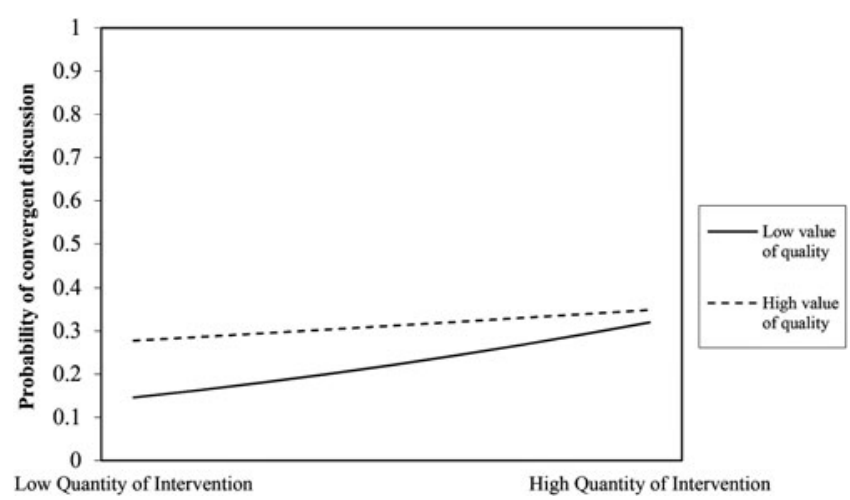

Figure 6. Interaction effect of quantity, moderated by quality, of HKFs intervention on convergent discussion (H9)* (quantity of intervention measured by number of HKFs' posts)

Notes: *Low value of moderator is defined as one s.d. below mean, and high value is defined as one s.d. above mean. Value of moderator is centered.

for stimulating divergent discussion, but another reason could be that the measure for quality of intervention should be more finely-grinded to observe any effects on online divergent thinking.

Finally, although we did not formally hypothesize the effect of HKFs' initiation (whether a discussion is initiated by an HKF), we find its effects on different KS outcomes as dependent variables interesting. It is consistently negatively related to the length of discussion (see results in Table 6) and to divergent thinking (see results in Table 9). A list of dependent and independent variables for the corresponding hypotheses with a summary of results are shown in Table 10.

\section{DISGUSSION}

\section{Theoretical Contribution and Practical Implications}

HKFs' online contributions, expressed through original posts or comments on others' posts, will be seen by all other community members. As a consequence, knowledge exchange in online communities (Li-Ying \& Salomo, 2013) is more likely to happen with the function of HKFs. The behavior of HKFs provides an additional clue for other community members, who can create a mental shortcut used as a judgment rule for making quick evaluations. As long as a number of other online community members (including other HKFs) react to an HKF's posts, a bandwagon effect can potentially emerge (Sundar, 2007; Sundar et al., 2008). As firms deploy HKFs as hidden moderators in their online KS communities, it is relevant and interesting to understand whether and how it works to foster greater KS. Informed and inspired by the insights gained from a number of indepth interviews with relevant stakeholders, we developed a number of hypotheses with regard to the relationships between the quantity and quality of HKFs' online contributions and community-level KS outcomes, and tested these hypotheses 

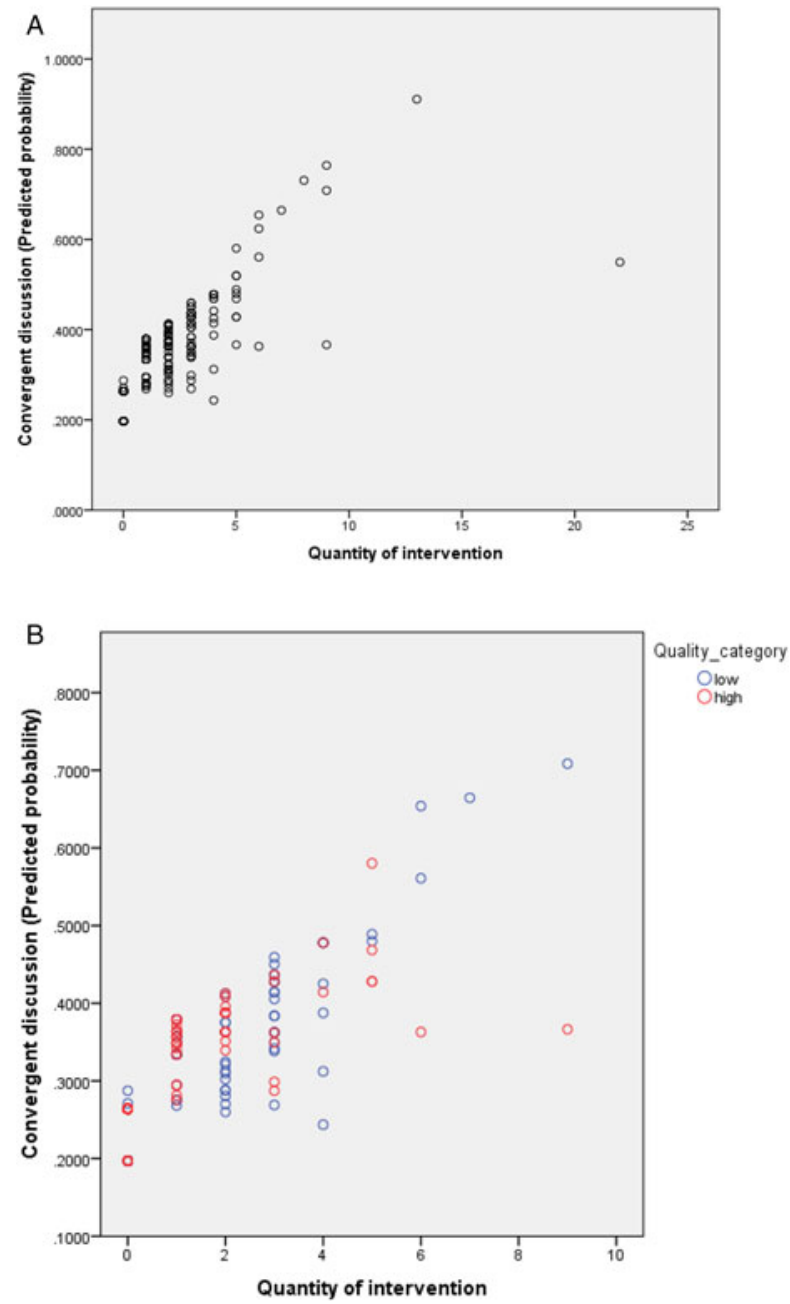

Figure 7A-7B. Scatterplot for effect of quantity, moderated by quality, of HKFs intervention on Convergent discussion (H9) (Quantity of intervention measured by number of HKFs' posts)

based on online log data using content analysis. The results reveal interesting and surprising findings.

First, companies need to understand that the role of HKFs and their effect on KS rely on both the quantity and quality of their contributions in the communities. To stimulate online interaction by extending the length of discussion, HKFs can either add more responses to a thread regardless of their quality, or to contribute to a thread with posts of high quality. However, it is not conclusive whether the approach of combining a large number of interventions with high quality posts will do good or harm to the intended outcome. Therefore, we suggest that the online community manager should guide HKFs to try out a mix of a relatively large number of posts with low quality and a relatively small number of posts with high quality.

Second, when a question is raised in an online discussion thread, it is important to pay attention to whether a solution is found in the discussion. This is an 
Table 9. Results of Binary Logistic Regression Models for H10, H11, and H12

\begin{tabular}{|c|c|c|c|c|}
\hline Dependent variables & $\begin{array}{l}\text { Divergent discussion } \\
\text { (Quantity of intervention } \\
\text { measured by number } \\
\text { of unique HKFs) }\end{array}$ & $\begin{array}{l}\text { Divergent discussion } \\
\text { (Quantity of intervention } \\
\text { measured by number } \\
\text { of unique HKFs) }\end{array}$ & $\begin{array}{l}\text { Divergent discussion } \\
\text { (Quantity of intervention } \\
\text { measured by number } \\
\text { of HKFs' posts) }\end{array}$ & $\begin{array}{l}\text { Divergent discussion } \\
\text { (Quantity of intervention } \\
\text { measured by number } \\
\text { of HKFs' posts) }\end{array}$ \\
\hline \multicolumn{5}{|l|}{ Independent variables } \\
\hline Constant & $-2.341(0.259)^{* * * *}$ & $-2.386(0.269)^{* * * *}$ & $-2.298(0.257)^{* * *}$ & $-2.331(0.264)^{* * * *}$ \\
\hline \multicolumn{5}{|l|}{ Control variables } \\
\hline $\begin{array}{l}\text { HKFs initiation } \\
\text { Month (March 2014) }\end{array}$ & $\begin{array}{l}-1.050(0.447)^{* *} \\
0.135(0.395)\end{array}$ & $\begin{array}{c}-1.075(0.450)^{* * *} \\
0.163(0.397)\end{array}$ & $\begin{array}{l}-1.387(0.515)^{* * * *} \\
0.052(0.410)\end{array}$ & $\begin{array}{l}-1.413(0.518)^{* * * *} \\
0.072(0.409)\end{array}$ \\
\hline \multicolumn{5}{|l|}{ Main effects } \\
\hline Quantity of intervention & $0.532(0.197)^{* * * *}($ odds ratio $=1.702)$ & $\begin{array}{c}0.642(0.247)^{* * *} \\
\text { (odds ratio }=1.899)\end{array}$ & $\begin{array}{c}0.306(0.104) * * * \\
(\text { odds ratio }=1.358)\end{array}$ & $\begin{array}{c}0.355(0.125) * * * \\
(\text { odds ratio }=1.427)\end{array}$ \\
\hline Quality of intervention & $0.803(0.602)$ & $0.704(0.611)$ & $\begin{array}{c}1.040(0.536)^{*} \\
(\text { odds ratio }=2.830)\end{array}$ & $\begin{array}{c}1.026(0.532)^{*} \\
\text { (odds ratio }=2.790)\end{array}$ \\
\hline Quality of intervention ${ }^{2}$ & $-0.266(0.208)$ & $-0.165(0.245)$ & $-0.326(0.194)^{*}$ & $-0.271(0.206)$ \\
\hline $\begin{array}{l}\text { Interaction effect } \\
\text { Quantity*Quality of intervention }{ }^{2}\end{array}$ & & $-0.061(0.084)$ & & $-0.036(0.051)$ \\
\hline \multicolumn{5}{|l|}{ Model statistics } \\
\hline Cox \& Snell $\mathrm{R}^{2}$ & 0.063 & 0.064 & 0.073 & 0.074 \\
\hline Hosmer and Lemeshow Test (df) & $4.701(6)$ & $4.485(6)$ & $3.006(6)$ & $1.226(5)$ \\
\hline
\end{tabular}

Notes: Significance levels: * $\mathrm{p}<0.10$; ** $\mathrm{p}<0.05 ; * * * \mathrm{p}<0.01 ;$ Standard errors in brackets; $\mathcal{N}=379$ 
Table 10. A summary of hypotheses and results

\begin{tabular}{|c|c|c|c|}
\hline Hypotheses & Dependent variable & Independent variable & Results \\
\hline $\mathrm{H} 1$ & $\begin{array}{l}\text { Length of } \\
\text { discussion }\end{array}$ & $\begin{array}{l}\text { Quantity of } \\
\text { intervention }\end{array}$ & Supported for both measures of quantity \\
\hline $\mathrm{H} 2$ & $\begin{array}{l}\text { Length of } \\
\text { discussion }\end{array}$ & Quality of intervention & Partially supported \\
\hline H3 & $\begin{array}{l}\text { Length of } \\
\text { discussion }\end{array}$ & $\begin{array}{l}\text { Quantity and quality of } \\
\text { intervention }\end{array}$ & $\begin{array}{l}\text { Not supported due to insignificant effect } \\
\text { size }\end{array}$ \\
\hline $\mathrm{H} 4$ & Solution found & $\begin{array}{l}\text { Quantity of } \\
\text { intervention }\end{array}$ & Supported for both measures of quantity \\
\hline H5 & Solution found & Quality of intervention & Supported for both measures of quantity \\
\hline H6 & Solution found & $\begin{array}{l}\text { Quantity and quality of } \\
\text { intervention }\end{array}$ & $\begin{array}{l}\text { Supported only for quantity measured by } \\
\text { number of HKFs' posts }\end{array}$ \\
\hline $\mathrm{H} 7$ & $\begin{array}{r}\text { Convergent } \\
\text { discucssion }\end{array}$ & $\begin{array}{l}\text { Quantity of } \\
\text { intervention }\end{array}$ & $\begin{array}{l}\text { Supported only for quantity measured by } \\
\text { number of HKFs' posts }\end{array}$ \\
\hline H8 & $\begin{array}{r}\text { Convergent } \\
\text { discucssion }\end{array}$ & Quality of intervention & Not supported \\
\hline H9 & $\begin{array}{r}\text { Convergent } \\
\text { discucssion }\end{array}$ & $\begin{array}{l}\text { Quantity and quality of } \\
\text { intervention }\end{array}$ & $\begin{array}{l}\text { Marginally supported only for quantity } \\
\text { measured by number of HKFs' posts }\end{array}$ \\
\hline $\mathrm{H} 10$ & $\begin{array}{l}\text { Divergent } \\
\text { discussion }\end{array}$ & $\begin{array}{l}\text { Quantity of } \\
\text { intervention }\end{array}$ & Supported for both measures of quantity \\
\hline H11 & $\begin{array}{l}\text { Divergent } \\
\text { discussion }\end{array}$ & Quality of intervention & $\begin{array}{l}\text { Marginally supported only for quantity } \\
\text { measured by number of HKFs' posts }\end{array}$ \\
\hline $\mathrm{H} 12$ & $\begin{array}{l}\text { Divergent } \\
\text { discussion }\end{array}$ & $\begin{array}{l}\text { Quantity and quality of } \\
\text { intervention }\end{array}$ & Not supported \\
\hline
\end{tabular}

indicator of effective $\mathrm{KS}$ among employees, because a discussion with a found solution will result in formalized and institutionalized knowledge that will be documented by the community manager on the knowledge bank portal. The results of our research in this regard show strong positive effects of quantity and quality of HKFs' intervention on the likelihood of solutions found. Moreover, a negative interactive effect of quantity and quality has a relatively small but sensible effect size. Therefore, we suggest that to ensure that a solution will be found in a discussion, HKFs may consider two possible strategies: (1) to get many HKFs to react to the question or to make many posts reacting to the question; and (2) to make high quality interventions. Using a large number of high quality interventions may not create higher likelihood of solutions found.

Third, convergent discussion can be fostered by a large number of posts made by $\mathrm{HKF}_{\mathrm{s}}$, or by involving many HKFs in a discussion thread. However, quality of HKFs' intervention does not necessarily help to form a convergent discussion online. The results of our research also show a negative interactive effect of quantity and quality with a marginal significance level and a small but sensible effect size. Therefore, we are confident to suggest that increasing the use of large number of posts with high quality may lower the chance of having convergent discussion in the online community.

Finally, if many HKFs make posts or if a large number of posts are made by a small number of HKFs, divergent discussion will probably emerge. In addition, a 
medium level of $\mathrm{HKF}$ intervention quality could be optimal to foster divergent discussion to some extent. In sum, the quantity and quality of HKFs' interventions present complex patterns of impact on KS outcome in online R\&D communities. Therefore, managers need to pay close attention to a balanced approach to using HKFs in terms of quantity and quality to foster KS, depending on the organizational objectives around KS.

Within the specific context of our research, these findings make clear contributions to the literature on KS with regard to how interventions made by knowledge facilitators can actually lead to desired KS outcomes for an organization. We suggest that future research make good use of KS practices in the digitalization process of organizations using content analysis for future empirical studies. In this way, our knowledge on the linkage among KS motivations, KS behavior, and KS outcomes can be enriched. These findings also make specific contributions to the understanding of the roles of knowledge facilitators in digitized organizations so that the typology proposed by Cabrera and Cabrera (2002) can be further developed.

Although using HKFs may be seen as an acceptable practice in China, other cultures may view it differently. This study is limited to the context of the business culture in China, where perceptions of what type of actions of an employer are acceptable by employees might be different from those in the Western cultures. As HKFs are hidden from regular employees, there is a question about whether regular employees will accept the practice of HKFs as a deliberate management decision. Prior research has suggested that perceived organizational justice and citizenship behavior differ between the US and China (Schilpzand, Martins, Kirkman, Lowe, \& Chen, 2013). Also, group harmony plays a significant role to mediate the effect of justice climate on group effectiveness (Ünal, Chen, \& Xin, 2017). Thus, the reason why HKFs can effectively facilitate online KS within an organization in China is deeply rooted in the Chinese culture. However, this does not mean that HKFs cannot be deployed in firms with Western culture at all if the practices of HKFs are well managed, as long as their organizational culture values group harmony and the good will of management more important than individual interests and justice. It can be interesting to see how firms in other national cultures perceive the usefulness of HKFs in online communities (Jackson, 2011). Here experimental design methods can be highly promising, for instance, to have two controlled business units of the same company (one in China and the other in the US) and test the potentially different effects of HKFs' KS intervention.

\section{Limitations and Future Research Directions}

This study has a number of limitations. First, it is based on a single case study using content analysis. We are aware that there are clearly limitations pertaining to a study based on the observation of a single organization (Yin, 1993). However, as the phenomenon of using HKFs within intrafirm online communities is likely a common practice among firms and their roles and effectiveness are theoretically

(C) 2018 The International Association for Chinese Management Research 
unnoticed and practically unclear, an in-depth study of such a kind is extremely helpful to explore the nature of HKFs, their interventions, and organizational implications. The combined use of pre-study interviews and online log data provide deep insight into a yet unexplored phenomenon. Should we have access to several similar case firms and replicate the research approach, the validity and generalizability of the findings will be significantly improved. Future study will benefit from a multi-case study design that takes cross-organizational differences into account. Second, in this study we only looked into the use of HKFs when they have already participated in the online R\&D communities and resumed their 'duties'. We were not able to investigate the behaviors of HKFs before they accepted and after they fulfilled their responsibilities. As participation and contribution to an online community is in a broad sense a special exchange relationship, future research in this direction may differentiate the roles of HKFs at different stages of involvement by observing HKFs' behaviors pre-exchange, during exchange, and after exchange (Li-Ying \& Salomo, 2013).

There is a limitation regarding the measures of divergent and convergent thinking. The binary measures that we use have an advantage of simplicity, but it might also miss out richer variation among situations in the real world. Alternative measures for divergent and convergent thinking are encouraged to be used in future research. Another limitation concerns the temporal effect of HKFs' online intervention on the occurrence of divergent and convergent thinking in online discussions. As the job mandate of HKFs in R\&D communities is to promote knowledge sharing and innovation, it is reasonable to expect that HKFs can use quantity as a means to inspire divergent discussion first, and then use quality as a means to help employees to converge the ideas to something constructive, tangible, and useful. Therefore, it could be highly interesting to develop hypotheses accordingly regarding the sequential effect of quantity and quality of HKFs' online intervention on divergent and convergent thinking, respectively. However, when measurements are only taken at the level of a discussion thread, involving multiple employees, it is practically very hard to examine such a potential sequential effect because, in fact, not all divergent and convergent thinking as cognitive processes in online community members' minds are manifested in the online discussion, and those offline actions of learning are not observable in this research. For instance, by observing the online discussion, we might only identify the occurance of divergent thinking among those who participated in the online discussion without identifying convergent discussions. However, this does not necessarily mean that some employees have not experienced some sort of intermediate process of convergent thinking and took some offline learning actions, which we could not observe. Conversely, if we could only observe convergent thinking manifested by online communication, this does not mean divergent discussions have not taken place offline. Therefore, it is very hard (and perhaps methodologically risky) to argue for the sequential effect and test it, if means of observing KS outcome is limited to only one form of communication. Future research may fill in this gap by using multiple mechanisms of observation. 


\section{Other Managerial Issues and Future Research}

First, firms using HKFs to intentionally influence employees in intrafirm online KS communities have a clear purpose: to transform a firm's organizational learning culture from a hierarchical to a distributed model. This is where theories of organizational culture become relevant to HKFs as well. Future research may draw on the literature on organizational culture - for instance, the three perspectives of organizational culture (Martin, 2002) and the group and grid culture theory (Jackson, 2011; Thompson, Ellis, \& Wildavosky, 1990) - to understand the rationale of using $\mathrm{HKF}_{\mathrm{s}}$ and the potential conflicts they might create. Future research may observe how a firm uses various means to achieve a transition of organizational culture without creating 'differentiation' and 'fragmentation' (Martin, 2002).

Second, the broad stream of the literature on management transparency has developed a dynamic perspective, which views transparency as an IT-driven communication process, in which firms and stakeholders interact to share information and cooperate (Santana \& Wood, 2009; Turilli \& Floridi, 2009). It is with this dynamic perspective that many large firms introduce intrafirm online KS platforms, because they want knowledge distributed among employees within the organization to be shared transparently without being constrained by the hierarchical organizational structure, creating an effective and efficient transactive memory system (Brandon \& Hollingshead, 2004). Role transparency ensures that the right persons with the right function and competence can enter the right kind of exchange relationship in a community (Nambisan, 2002), and process transparency makes information exchange relationships clear and explicit (Nambisan, 2002; West \& O’Mahony, 2008). The problem of HKFs' actions in intrafirm online $\mathrm{KS}$ communities is that role transparency and process transparency might be compromised. Future research is encouraged to investigate the impact of predefined role and process transparency on the perceived roles and actual actions of HKFs in an online KS community, which may in turn reshape the transparency of the online community as a whole.

Last but not least, the fact that HKFs' identities and roles are non-transparent to regular community members may raise the issue of management ethics (Sandin, 2009). Some empirical studies have suggested that an ethical culture is needed for successful adoption of information systems (Ruppel \& Harrington, 2001) and ethical leadership is important as well because it influences followers' decisionmaking (Steinbauer, Renn, Taylor, \& Njoroge, 2014). At first glance, the deployment of HKFs is far from the virtue of truthfulness, a higher level of management transparency (das Neves \& Vaccaro, 2013). However, the literature has also recognized that there are not only ethics of justice but also ethics of care (Sandin, 2009; Simola, 2003, 2005): HKFs might not be regarded as ethical in justice, but they can be used to strengthen ethics of care for the sake of fostering better organizational culture and enhancing KS by judiciously responding to other people's posts about new ideas and threads, maintaining the active atmosphere within a community, and continuously motivating participants' contribution. Therefore, 
the behaviors of HKFs serve the organization, an important aspect in management ethics (Jackson, 2000; 2001). On the other hand, once the systematic use of HKFs is compromised (i.e., HKFs are detected by regular online community members), it may completely backfire, and senior management may be criticized for taking advantage of employees' trust and manipulating their knowledge contribution. Future research should observe management and HKFs in these circumstances in a well-defined context.

\section{GONGLUSION}

Although we believe that HKFs are often used in the KS online platforms of large companies, the unique phenomenon of HKFs is still far from being well understood. Primarily based on the literature of KS and KS facilitation, we explore how HKFs make their online interventions in terms of quantity and quality to achieve desired KS outcomes in online R\&D communities. Both quantity and quality of HKFs' online contribution have impacts on the KS outcomes of online communities in a complex and interactive fashion, depending on the objectives of KS outcomes. Senior managers interested in using HKFs should consider the findings of this study carefully, to ensure effective enhancement of KS and innovation within their organizations.

\section{REFERENGES}

Adendorff, D. E. 2005. An investigation into the roles and competencies of an online facilitator (Doctoral dissertation). University of Pretoria.

Ardichvili, A. 2008. Learning and knowledge sharing in virtual communities of practice: Motivators, barriers, and enablers. Advances in Developing Human Resources, 10(4): 541-554.

Asch, S. E. 1955. Opinions and social pressure. Scientific American, 193(5): 31-35.

Banerjee, A. V. 1992. A simple model of herd behavior. The Quarterly Joumal of Economics, 107(3): 797-817.

Baron, R. S., Vandello, J. A., \& Brunsman, B. 1996. The forgotten variable in conformity research: Impact of task importance on social influence. Journal of Personality and Social Psychology, 7 1(5): 915-927.

Basadur, M., Runco, M. A., \& Vega, L. 2000. Understanding how creative thinking skills, attitudes and behaviors work together: A causal process model. The Journal of Creative Behavior, 34 (2): $77-100$.

Berger, J., \& Heath, C. 2007. Where consumers diverge from others: Identity signaling and product domains. Journal of Consumer Research, 34(2): 121-134.

Bikhchandani, S., Hirshleifer, D., \& Welch, I. 1998. Learning from the behavior of others: Conformity, fads, and informational cascades. Journal of Economic Perspectives, 12(3): $151-170$.

Bliss, C. A., \& Lawrence, B. 2009. From posts to patterns: A metric to characterize discussion board activity in online courses. Journal of Asynchronous Learning Networks, 13(2): 15-32.

Bordia, P., Irmer, B. E., \& Abusah, D. 2006. Differences in sharing knowledge interpersonally and via databases: The role of evaluation apprehension and perceived benefits. European Journal of Work and Organizational Psychology, 15(3): 262-280.

Brandon, D. P., \& Hollingshead, A. B. 2004. Transactive memory systems in organizations: Matching tasks, expertise, and people. Organization Science, 15(6): 633-644.

Cabrera, A., \& Cabrera, E. F. 2002. Knowledge-sharing dilemmas. Organization Studies, 23(5): $687-710$. 
Cabrera, A., Collins, W. C., \& Salgado, J. F. 2006. Determinants of individual engagement in knowledge sharing. International Journal of Human Resource Management, 17(2): 245-264.

Cacciamani, S., Cesareni, D., Martini, F., Ferrini, T., \& Fujita, N. 2012. Influence of participation, facilitator styles, and metacognitive reflection on knowledge building in online university courses. Computers \& Education, 58(3): 874-884.

Cameron, C., \& Trivedi, P. 2005. Microeconometrics: Methods and applications. Cambridge: Cambridge University Press.

Cesareni, D., Albanese, O., Cacciamani, S., Castelli, S., De Marco, B., Fiorilli, C., Luciani, M., Mancini, I., Martini, F., \& Vanin, L. 2008. Tutorship styles and knowledge building in an online community: Cognitive and metacognitive aspects. In B. M. Varisco (Ed.), Psychological, pedagogical and sociological models for learning and assessment in virtual communities: 13-56. Milano, Italy: Polimetrica International Scientific Publisher.

Chen, C. J., \& Hung, S. W. 2010. To give or to receive? Factors influencing members' knowledge sharing and community promotion in professional virtual communities. Information \& Management, 47(4): 226-236.

Cook, S. 2008. The contribution revolution: Letting volunteers build your business. Harvard Business Revieze, 86(10): 60-69.

Corneo, G., \& Jeanne, O. 1997. Snobs, bandwagons, and the origin of social customs in consumer behavior. Joumal of Economic Behavior and Organization, 32(3): 333-347.

Correia, A. P., \& Baran, E. 2010. Lessons learned on facilitating asynchronous discussions for online learning. Educação, Formação \& Tecnologias, 3(1): 59-67. ISSN 1646-933X

Cropley, A. 2006. In praise of convergent thinking. Creativity Research Journal, 18(3): 391-404.

Cummings, J. N. 2004. Work groups, structural diversity, and knowledge sharing in a global organization. Management Science, 50(3): 352-364.

Dahlander, L., \& Magnusson, M. G. 2005. Relationships between open source software companies and communities: Observations from Nordic firms. Research Policy, 34(4): 481-493.

Dahlander, L., \& Magnusson, M. G. 2008. How do firms make use of open source communities? Long Range Planning, 41(6): 629-649.

Damodaran, L., \& Olphert, W. 2000. Barriers and facilitators to the use of knowledge management systems. Behaviour \& Information Technology, 19(6): 405-413.

das Neves, J. C., \& Vaccaro, A. 2013. Corporate transparency: A perspective from Thomas Aquinas' summa theologiae. Joumal of Business Ethics, 113(4): 639-648.

Dijksterhuis, A., \& Meurs, T. 2006. Where creativity resides: The generative power of unconscious thought. Consciousness and Cognition, 15(1): 135-146.

Dutton, W. H. 2008. The wisdom of collaborative network organizations: Capturing the value of networked individuals. Prometheus, 26(3): 211-230.

Edmunds, A., \& Morris, A. 2000. The problem of information overload in business organisations: A review of the literature. International Journal of Information Management, 20(1): 17-28.

Eppler, M. J., \& Mengis, J. 2004. The concept of information overload: A review of literature from organization science, accounting, marketing, MIS, and related disciplines. The Information Society, 20(5): 325-344.

Escalas, J. E., \& Bettman, J. R. 2005. Self-construal, reference groups, and brand meaning.Jourmal of Consumer Research, 32(3): 378-389.

Gagné, M. 2009. A model of knowledge--sharing motivation. Human Resource Management, 48 (4): 571-589.

Gilbert, P. K., \& Dabbagh, N. 2005. How to structure online discussions for meaningful discourse: A case study. British Journal of Educational Technology, 36(1): 5-18.

Grandori, A. 1997. Governance structures, coordination mechanisms and cognitive models.Journal of Management and Governance, 1(1): 29-47.

Greve, H. R. 2018. Show us the data! Improving evidence presentation for publication. Management and Organization Revieze, 14(2): 423-432.

Haas, M. R., \& Hansen, M. T. 2005. When using knowledge can hurt performance: The value of organizational capabilities in a management consulting company. Strategic Management Journal, 26(1): 1-24.

Haas, M. R., \& Hansen, M. T. 2007. Different knowledge, different benefits: Toward a productivity perspective on knowledge sharing in organizations. Strategic Management Journal, 28(11): $1133-1153$.

Hackman, J. R. 1987. The design of work teams. In J. Lorsch (Ed.), Handbook of organizational behavior: 315-342. Englewood Cliffs, NJ: Prentice-Hall. 
Hommel, B., Colzato, L. S., Fischer, R., \& Christoffels, I. K. 2011. Bilingualism and creativity: Benefits in convergent thinking come with losses in divergent thinking. Bilingualism and Cognitive Control, $111(2)$ : article 273.

Hsu, M. H., Ju, T. L., Yen, C. H., \& Chang, C. M. 2007. Knowledge sharing behavior in virtual communities: The relationship between trust, self-efficacy, and outcome expectations. International Journal of Human-Computer Studies, 65(2): 153-169.

Huang, J.-H., \& Chen, Y.-F. 2006. Herding in online product choice. Psychology \& Marketing, 23(5): 413-428.

Jackson, T. 2000. Management ethics and corporate policy: A cross-cultural comparison.Jourmal of Management Studies, 37(3): 349-368.

Jackson, T. 2001. Cultural values and management ethics: A 10-nation study. Human Relations, 54(10): 1267-1302.

Jackson, S. E., Chuang, C.-H., Harden, E. E., Jiang, Y., \& Joseph, J. M. 2006. Toward developing human resource management systems for knowledge-intensive teamwork. In J. M. Joseph (Ed.), Research in personnel and human resources management, 25: 27-70. Amsterdam, Germany: JAI.

Jackson, S. 2011. Organizational culture and information systems adoption: A three-perspective approach. Information and Organization, 21(2): 57-83.

Jauk, E., Benedek, M., \& Neubauer, A. C. 2012. Tackling creativity at its roots: Evidence for different patterns of EEG alpha activity related to convergent and divergent modes of task processing. International Journal of Psychophysiology, 84(2): 219-225.

Jeppesen, L. B., \& Frederiksen, L. 2006. Why do users contribute to firm-hosted user communities? The case of computer-controlled music instruments. Organization Science, 17(1):45-63.

Kardes, F. R., Posavac, S. S., \& Cronley, M. L. 2004. Consumer inference: A review of processes, bases, and judgment contexts. Journal of Consumer Psychology, 14(3): 230-256.

Kozinets, R.V. 1998. On netnography: Initial reflections on consumer research investigations of cyberculture. Advance in Consumer Research, 25(1): 366-372.

Kulkarni, U. R., Ravindran, S., \& Freeze, R. 2006. A knowledge management success model: Theoretical development and empirical validation. Journal of Management Information Systems, 23(3): 309-347.

Lee, J.-N. 2001. The impact of knowledge sharing, organizational capability and partnership quality on IS outsourcing success. Information and Management, 38(5): 323-335.

Lee, H., \& Choi, B. 2003. Knowledge management enablers, processes, and organizational performance: An integrative view and empirical examination. Journal of Management Information Systems, 20(1): 179-228.

Leibenstein, H. 1950. Bandwagon, snob, and Veblen effects in the theory of consumers' demand. The Quarterly Joumal of Economics, 64(2): 183-207.

Levine, S. S. 2018. Show us your data: Connect the dots, improve science. Management and Organization Revieze, 14(2): 433-437.

Li-Ying, J., \& Salomo, S. 2013. Design of governance in virtual communities: Definition, mechanisms, and variation patterns. International Journal of Collaborative Enterprises, 3(4): 225-251.

Ma, W. W., \& Yuen, A. H. 2011. Understanding online knowledge sharing: An interpersonal relationship perspective. Computers \& Education, 56(1): 210-219.

Masters, K., \& Oberprieler, G. 2004. Encouraging equitable online participation through curriculum articulation. Computers \& Education, 42(4): 319-332.

Martin, J. 2002. Organizational culture: Mapping the terrain. Thousand Oaks, CA: Sage Publications.

Naim, M. F., \& Lenka, U. 2017. Linking knowledge sharing, competency development, and affective commitment: Evidence from Indian Gen Y employees. Journal of Knozeledge Management, online early view, https://doi.org/10.1108/JKM-08-2016-0334.

Nambisan, S. 2002. Designing virtual customer environments for new product development: Toward a theory. Academy of Management Revieze, 27(3): 392-413.

Nambisan, S., \& Baron, R. A. 2007. Interactions in virtual customer environments: Implications for product support and customer relationship management.Journal of Interactive Marketing, 21(2): 42-62.

Nandi, D., Hamilton, M., \& Harland, J. 2012. Evaluating the quality of interaction in asynchronous discussion forums in fully online courses. Distance Education, 33(1): 5-30.

Nisbet, D. 2004. Measuring the quantity and quality of online discussion group interaction. Journal of eLiteracy, 1(2): 122-139. 
Pee, L. G., Pee, L. G., Min, J., \& Min, J. 2017. Employees' online knowledge sharing: the effects of person-environment fit. Journal of Knozledge Management, 21(2): 432-453.

Reinholt, M. I. A., Pedersen, T., \& Foss, N. J. 2011 . Why a central network position isn't enough: The role of motivation and ability for knowledge sharing in employee networks. Academy of Management Journal, 54(6): 1277-1297.

Runco, M. A. 2007. Creativity: Theories and themes: Research, development, and practice. New York: Academic Press.

Runco, M. A., \& Acar, S. 2012. Divergent thinking as an indicator of creative potential. Creativity Research Journal, 24(1): 66-75.

Ruppel, C. P., \& Harrington, S. J. 2001. Sharing knowledge through intranets: A study of organizational culture and intranet implementation. IEEE Transactions on Professional Communication, 44(1): 37-52.

Salmon, G. 2000. E-Moderating - The key to teaching and learning online. London, UK: Kogan Page.

Sandin, P. 2009. Approaches to ethics for corporate crisis management. Jourmal of Business Ethics, 87(1): 109-116.

Santana, A., \& Wood, D. 2009. Information vs. knowledge: Transparency and social responsibility issues for Wikipedia. Ethics and Information Technology, 11(2): 133-144.

Schilpzand, M., Martins, L. L., Kirkman, B. L., Lowe, K. B., \& Chen, Z. X. 2013. The relationship between organizational justice and organizational citizenship behavior: The moderating role of cultural value orientation. Management and Organization Revieze, 9(2): 345-374.

Shah, S. K. 2006. Motivation, governance, and the viability of hybrid forms in open source software development. Management Science, 52(7): 1000-1014.

Simola, S. 2003. Ethics of justice and care in corporate crisis management. Journal of Business Ethics, 46(4): 351-361.

Simola, S. 2005. Concepts of care in organizational crisis prevention.Journal of Business Ethics, 62(4): 341-353.

Steinbauer, R., Renn, R. W., Taylor, R. R., \& Njoroge, P. K. 2014. Ethical leadership and followers' moral judgment: The role of followers' perceived accountability and self-leadership. Journal of Business Ethics, 120(3): 381-392.

Sundar, S. S. 2007. The MAIN Model: A heuristic approach to understanding technology effects on credibility. In M. J. Metzger \& A. J. Flanagin (Eds.), Digital media, youth, and credibility: 73-100. The John D. and Catherine T. MacArthur Foundation Series on Digital Media and Learning. Cambridge, MA: The MIT Press.

Sundar, S. S., Oeldorf-Hirsch, A., \& Xu, Q. 2008. The bandwagon effect of collaborative filtering technology. CHI conference proceedings, Florence, Italy.

Sundaramurthy, C., \& Lewis, M. 2003. Control and collaboration: Paradoxes of governance. Academy of Management Revieze, 28(3): 397-415.

Taylor, W. A., \& Wright, G. H. 2004. Organizational readiness for successful knowledge sharing: Challenges for public sector managers. Information Resources Management Journal, 17(2): 22-37.

Thompson, M., Ellis, R., \& Wildavosky, A. 1990. Culture theory. Boulder, CO: Westview Press.

Turilli, M., \& Floridi, L. 2009. The ethics of information transparency. Ethics and Information Technology, 11(2): 105-112.

Ünal, A., Chen, C., \& Xin, K. 2017. Justice climates and management team effectiveness: The central role of group harmony. Management and Organization Revieze, 13(4): 821-849.

van Herpen, E., Pieters, R., \& Zeelenberg, M. 2009. When demand accelerates demand: Trailing the bandwagon. Journal of Consumer Psychology, 19(3): 302-312.

von Hippel, E., \& von Krogh, G. 2003. Open source software and the 'private-collective' innovation model: Issues for organization science. Organization Science, 14(2): 209-223.

Walsh, J. P., \& Seward, J. K. 1990. On the efficiency of internal and external corporate control mechanisms. Academy of Management Revieze, 15(3): 421-458.

Wang, S., \& Noe, R. A. 2010. KS: A review and directions for future research. Human Resource Management Revieze, 20(2): 115-131.

Wasko, M. M., \& Faraj, S. 2005. Why should I share? Examining social capital and knowledge contribution in electronic networks of practice. MIS Quarterly, 29(1): 35-57.

Wellman, B., Salaff, J., Dimitrova, D., Garton, L., Gulia, M., \& Haythornthwaite, C. 1996. Computer networks as social networks: Collaborative work, telework, and virtual community. Annual Revieze of Sociology, 22(1): 213-238. 
West, J., \& O'Mahony, S. 2008. The role of participation architecture in growing sponsored open source communities. Industry and Innovation, 15(2): 145-168.

Westerski, A., Dalamagas, T., \& Iglesias, C. A. 2013. Classifying and comparing community innovation in Idea Management Systems. Decision Support Systems, 54(3): 1316-1326.

Wickersham, L. E., \& Dooley, K. E. 2006. A content analysis of critical thinking skills as an indicator of quality of online discussion in virtual learning communities. Quarterly Revieze of Distance Education, 7(2): 185.

Wise, K., Hamman, B., \& Thorson, K. 2006. Moderation, response rate, and message interactivity: Features of online communities and their effects on intent to participate. Journal of Computer-Mediated Communication, 12(1): 24-41.

Yin, R. 1993. Applications of case study research. Beverley Hills, CA: Sage Publishing.

Jason Li-Ying (yinli@dtu.dk) is professor at the Technology and Innovation Management division of DTU department of Management Engineering, Technical University of Denmark. Jason has research interests in technology and innovation management, organizational learning, and strategic management. His work has been published in scientific journals such as Long Range Planning, Fournal of Product Innovation Management, Asia Pacific Fournal of Management, Technovation, Technological Forecasting and Social Change, RED Management, Journal of Knowledge Management, etc. Jason is also an expert committee member for the Danish Ministry of Education and Research in the economics and business disciplines.

Zhinan Zhang (zhinanz@sjtu.edu.cn) is associate professor in the School of Mechanical Engineering, Shanghai Jiao Tong University. He received his $\mathrm{PhD}$ in 2011 from Shanghai Jiao Tong University, Shanghai, China. After that he was a post doctor in Shanghai Jiao Tong University and a visiting scholar at Technical University of Denmark. His research interests include theory and methods of design engineering and innovation, and computational design and analysis of tribosystems.

Qing Long (longqing1127@163.com) is a professional in intellectual property business of Kuangzhen Law Group at Hunan, China. He received his bachelor's degree from Chongqing University, China. After that he was a research engineer in the field of Mechanical engineering, online community manager of a large Chinese industry machinery corporation, and a lecturer at Hunan Land Enterprise Consulting Company Now.

Manuscript received: May 26, 2016

Final version accepted: September 3, 2018 (number of revisions - 4)

Accepted by: $\quad$ Senior Editor Zhi-Xue Zhang 